\title{
The Petrology of the Tholeiites through Melilite Nephelinites on Gran Canaria, Canary Islands: Crystal Fractionation, Accumulation, and Depths of Melting
}

\author{
by KAJ HOERNLE ${ }^{1}$ AND HANS-ULRICH SCHMINCKE \\ ${ }^{1}$ Earth Sciences, University of California, Santa Cruz, California 95064 \\ ${ }^{2}$ GEOMAR, Ableilung Vulkanologie, Wischhofstr. 1-3, D-2300 Kiel-14, Germany
}

(Received 14 October 1990; revised typescript accepted 6 October 1992)

\begin{abstract}
We report major and trace element X-ray fluorescence (XRF) data for mafic volcanics covering the 15-Ma evolution of Gran Canaria, Canary Islands. The Miocene (12-15 Ma) and Pliocene-Quaternary (0-6 Ma) mafic volcanics on Gran Canaria include picrites, tholeiites, alkali basalts, basanites, nephelinites, and melilite nephelinites. Olivine \pm clinopyroxene are the major fractionating or accumulating phases in the basalts. Plagioclase, $\mathrm{Fe}-\mathrm{Ti}$ oxide, and apatite fractionation or accumulation may play a minor role in the derivation of the most evolved mafic volcanics. The crystallization of clinopyroxene after olivine and the absence of phenocrystic plagioclase in the Miocene tholeiites and in the Pliocene and Quaternary alkali basalts and basanites with $\mathrm{MgO}>6$ suggests that fractionation occurred at moderate pressure, probably within the upper mantle. The presence of plagioclase phenocrysts and chemical evidence for plagioclase fractionation in the Miocene basalts with $\mathrm{MgO}<6$ and in the Pliocene tholeiites is consistent with cooling and fractionation at shallow depth, probably during storage in lower-crustal reservoirs. Magma generation at pressures in excess of 3.0-3.5 GPa is suggested by (a) the inferred presence of residual garnet and phlogopite and (b) comparison of $\mathrm{FeO}^{t}$ cation mole percentages and the CIPW normative compositions of the mafic volcanics with results from high-pressure melting experiments. The Gran Canaria mafic magmas were probably formed by decompression melting in an upwelling column of asthenospheric material, which encountered a mechanical boundary layer at $\sim 100-\mathrm{km}$ depth.
\end{abstract}

\section{INTRODUCTION}

The Canary Islands (Spain) form a 600-km chain of seven volcanic islands (Fig. 1) off the coast of southern Morocco. The age progression for the inception of volcanism is roughly from east (30-80 Ma) to west (2-4 Ma) (McDougall \& Schmincke, 1976; Feraud et al., 1985; Le Bas et al., 1986b). The geochemical and morphologic evolution of some of the Canary volcanoes is similar to that of other ocean island volcanoes such as those in the Hawaiian chain (Schmincke, 1973, 1982). To place constraints on the origin of the diverse suite of basalts on Gran Canaria, the third most easterly island, and ultimately on models for the origin of the Canaries, we have undertaken a detailed study of the major and trace elements (in this paper and in a companion paper, Hoernle \& Schmincke, this volume), and Sr, Nd, and $\mathrm{Pb}$ isotopes (Hoernle et al., 1991) of the mafic volcanics on Gran Canaria.

We chose to study Gran Canaria for several reasons: (1) the island is centrally located in the chain; (2) the mafic volcanics range widely in chemical composition, from picrite and tholeiite to alkali basalt to basanite to nephelinite, including melilite-bearing nephelinite; (3) tholeiite-nephelinite suites are present in both the Miocene and the Pliocene cycles of 

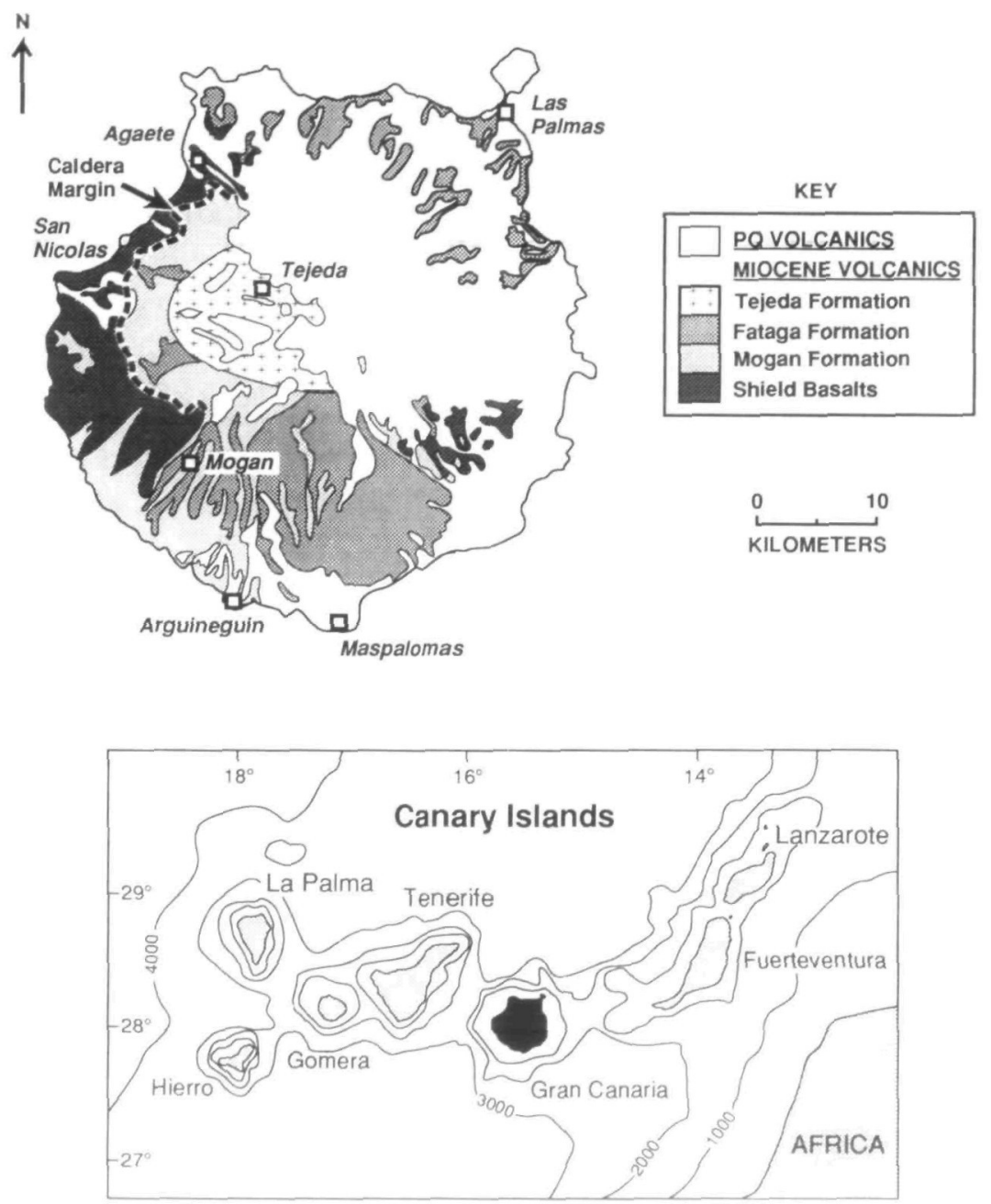

FIG. 1. Geological map of Gran Canaria with an inset map of the Canary Islands modified from Schmincke (1976). PQ-Pliocene-Quaternary.

volcanism; (4) deep radial canyons provide both relatively fresh samples and excellent stratigraphic control; (5) there have been several K/Ar age-dating studies (Abdel-Monem et al., 1971; Lietz \& Schmincke, 1975; McDougall \& Schmincke, 1976; Feraud et al., 1981; Schmincke et al., in prep.) and some Ar/Ar age-dating studies (van den Bogaard et al., 1988); and (6) there are systematic variations in composition and eruption rates as a function of age. Because of the extremely wide compositional range of magmas erupted from a single volcano, this study has implications for the petrogenesis of mafic magmas.

Although some of the Miocene and Pliocene-Quaternary mafic volcanics with $>44 \mathrm{wt} . \%$ $\mathrm{SiO}_{2}$ show evidence of slight lithospheric contamination, the isotopic data are consistent with all of the mafic magmas from a given age group (i.e., Miocene and Pliocene-Quaternary) being derived from similar source material (Hoernle et al., 1991). Therefore the differences in major and trace element concentrations between the mafic volcanics in an age group primarily reflect differences in melting conditions, such as temperature, pressure, and volatile content, and fractionation and accumulation processes during transport to the 
surface. The objectives of this paper are (1) to present the major and trace element [X-ray fluorescence (XRF) and instrumental neutron activation analysis (INAA)] data for the diverse suite of mafic volcanics on Gran Canaria, (2) to determine which, if any, of the mafic volcanics represent primary or near-primary compositions, (3) to evaluate the role of crystal fractionation in generating the non-primary mafic volcanics, (4) to place constraints on the depths at which the mafic magmas cooled and fractionated, and (5) to define the depths (pressures) of melting at which the mafic magmas were generated. In the companion paper in this volume, we will evaluate (1) the role of partial melting in generating some of the major and trace element variations, (2) differences in the major and trace element composition of the Miocene and Pliocene-Quaternary sources, and (3) the geochemical evolution of the Gran Canaria sources over the past $15 \mathrm{Ma}$.

\section{GENERAL GEOLOGY}

The subaerial eruptive history of Gran Canaria can be divided into three cycles (Fig. 2): a Miocene Cycle (8.5-15 Ma), a late Miocene through Pliocene Cycle (1.8-6.0 Ma), which we will simply refer to as the Pliocene Cycle hereafter, and a Quaternary Cycle $(0-1.8 \mathrm{Ma})$ (Schmincke, 1976, 1982, 1990). Each cycle on Gran Canaria has an evolution similar to that proposed for a typical Hawaiian volcano. The Hawaiian pre-shield, shield, post-shield (or post-caldera), and rejuvenated (or post-erosional) stages are contained within a single Gran Canaria cycle of volcanism (Fig. 2). The initial stage of the Miocene Cycle is not subaerially exposed on Gran Canaria. The oldest subaerially exposed volcanics of the Miocene Cycle, belonging to the Guigui, Hogarzales, and Mogan Formations (13.4-15 Ma), range in composition from picrites and tholeiites to peralkaline rhyolites, with the overall degree of differentiation increasing up section. The Fataga and Tejeda volcanics and intrusives (8.6-13.4 Ma) consist predominantly of trachyte and phonolite, with rare basanite and nephelinite dikes and flows. In summary, the mafic volcanics and calculated parental compositions for the evolved volcanics become more $\mathrm{SiO}_{2}$-undersaturated up section. The recharge or magma production rate (which equals the eruption rate corrected for volume loss as a result of crystal fractionation) also decreased with decreasing age (Schmincke, 1982; Crisp, 1984; van den Bogaard et al., 1988; Hoernle \& Schmincke, this volume).

An $\sim 3$-Ma volcanic hiatus and period of erosion separates the Miocene from the Pliocene Cycle (Fig. 2). The evolution of the Pliocene Cycle is considerably more complex than that of the Miocene Cycle. The oldest dated unit from the Pliocene Cycle is a sequence of tholeiitic pahoehoe flows belonging to the El Tablero Formation. Sample B14 from this sequence produced a whole-rock K/Ar age of $5.7 \pm 0.5 \mathrm{Ma}$ (H.-U. Schmincke, unpub. data). This tholeiitic unit is under- and overlain by alkali basalt and basanite lava flows. In addition, basanite, nephelinite, hawaiite, and tephrite flows from the El Tablero Formation have yielded K/Ar whole-rock ages of > 5.6-5.0 Ma (Lietz \& Schmincke, 1975; McDougall \& Schmincke, 1976; Feraud et al., 1981). In summary, the early part of the Pliocene Cycle ( 6-5 Ma) is characterized by a wide range of mafic volcanics (nephelinite through tholeiite, with rare hawaiite and tephrite), erupted from vents scattered across the island.

In contrast to the El Tablero Formation, the Roque Nublo Group ( 4.9-3.2 Ma) contains a limited range of mafic volcanics, but a wide range of evolved volcanics and intrusives. The Roque Nublo volcanics consist of complete suites of alkali basalt through trachyte and basanite through phonolite. Nephelinites are absent and tholeiites are rare. Rocks within a suite can be related through crystal fractionation processes. With decreasing age during the Roque Nublo, there was a decrease in (1) $\mathrm{SiO}_{2}$ saturation of the mafic 


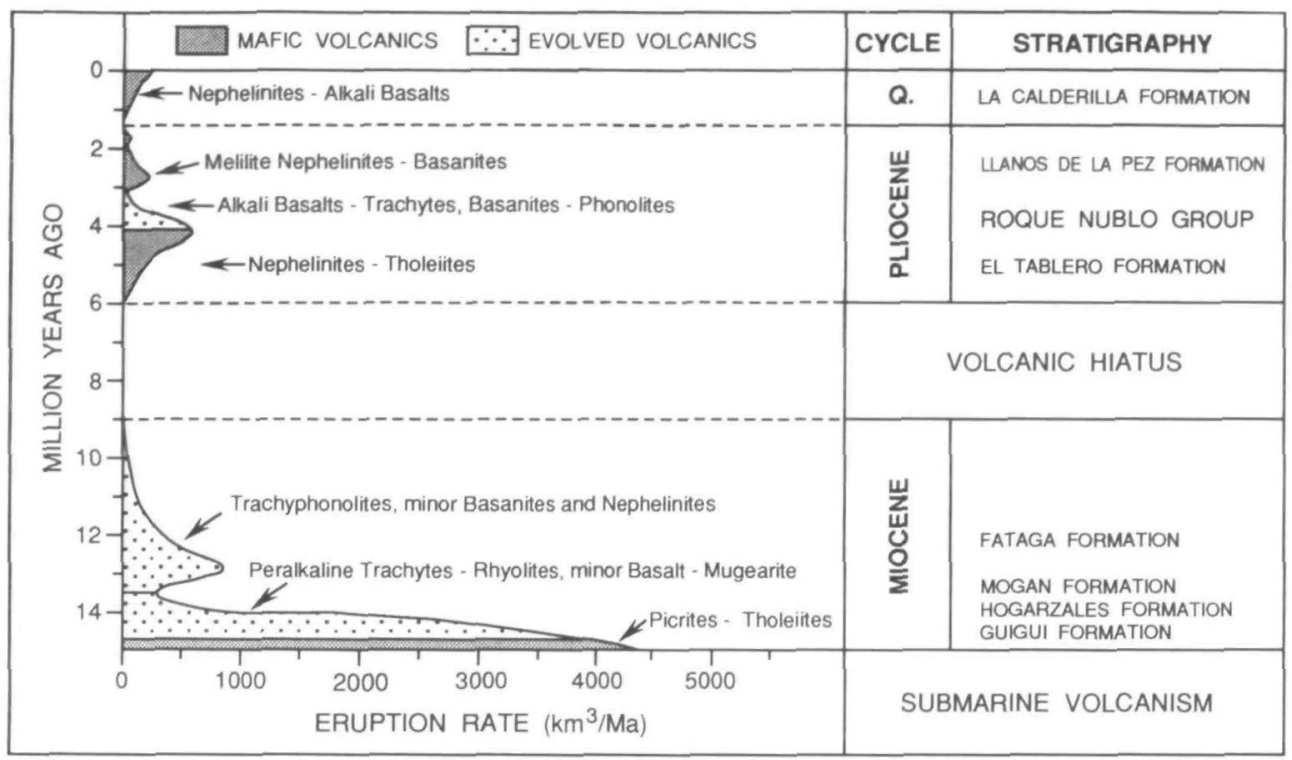

FIG. 2. Eruption rate vs. age for the Miocene, Pliocene, and Quaternary subaerial cycles of volcanism on Gran Canaria. Heavy stippled pattern denotes predominantly mafic $(m g$-number $\geqslant 62)$ volcanism, whereas the light stippled pattern denotes periods of predominantly evolved $(m g$-number $<62)$ volcanism. The Miocene Cycle makes up $\sim 80 \%$ of the subaerial volume, the Pliocene Cycle $\sim 18 \%$, and the Quaternary Cycle $\sim 2 \%$. A Gran Canaria cycle contains the pre-shield, shield, post-shield, and rejuvenated stages of a Hawaiian volcano.

volcanics and of the estimated parental compositions for evolved volcanics and (2) eruption rates and calculated magma production rates, but an overall increase in the degree of differentiation (Brey \& Schmincke, 1980; Hoernle, 1987; Hoernle \& Schmincke, this volume).

The Llanos de la Pez Formation ( $3 \cdot 2-1 \cdot 8 \mathrm{Ma})$ stratigraphically overlies the Roque Nublo Group. Although the contact between the two is unconformable in many locations, recent data suggest that these formations are not separated by a volcanic hiatus as previously proposed (Lietz \& Schmincke, 1975; McDougall \& Schmincke, 1976). The oldest dated Llanos de la Pez flows, both nephelinites, are 3.1 Ma (McDougall \& Schmincke, 1976; Schmincke et al., in prep.). An alkali basalt and mugearite from northern Gran Canaria, which have geochemical characteristics of the Roque Nublo Group, have ages of $3.15 \mathrm{Ma}$ and 2.91 Ma, respectively (Schmincke et al., in prep.). Nephelinite flows under- and overlie a phonolite tuff in Barranco de Guayadeque, and at several locations evolved dikes intrude nephelinite flows and are intruded by nephelinite dikes. Further evidence against a volcanic hiatus comes from the La Fortaleza section near Santa Lucia. Two Roque Nublo flows, a tephrite and the immediately overlying tephritic phonolite, yielded ages of $3.6 \mathrm{Ma}$ and 3.5 Ma, respectively [Abdel-Monem et al. (1971) dated the tephrite; McDougall \& Schmincke (1976) dated both flows]. These flows are reversely magnetized (H.-U. Schmincke \& U. Bleil, unpub. data) and are overlain by several thick volcanic breccias, common in the upper Roque Nublo Group. More than seven nephelinite flows overlie the breccias but underlie one of the nephelinite flows dated at 3.1 Ma by McDougall \& Schmincke. The dated flow is reversely magnetized yet the stratigraphically lower nephelinites are normally magnetized (H.-U. Schmincke \& U. Bleil, unpub. data) and thus must have ages between 3.15 and 3.4 Ma (Mankinen \& Dalrymple, 1979). Therefore the 
boundary between the Roque Nublo and Llanos de la Pez primarily reflects a change in chemistry to more mafic and more $\mathrm{SiO}_{2}$-undersaturated volcanics, rather than a hiatus in volcanism. Erosional rates were particularly high during late Roque Nublo volcanism, as evidenced by abundant vegetation imprints, conglomerates, and intragroup canyons.

Primitive nephelinite is the most abundant rock type in the Llanos de la Pez, followed by primitive basanite and melilite nephelinite. Compositions with $m g$-number $<61$ are rare. Although most of the melilite nephelinites stratigraphically overlie the nephelinite flows (Schmincke, 1976, 1990), consistent with the age of $1.8 \mathrm{Ma}$ obtained from a melilite nephelinite dike (McDougall \& Schmincke, 1976), at least one melilite nephelinite at Los Pechos was erupted during the early nephelinitic volcanism. Melilite nephelinite sample 1112 came from a flow which is five flows beneath a nephelinite flow dated at $2.8 \mathrm{Ma}$ (sample 1118 ; Lietz \& Schmincke, 1975).

Nephelinites were erupted during the early Quaternary (1-1.8 Ma), whereas the more recent volcanics $(0-1 \mathrm{Ma})$ consist primarily of basanite with rare alkali basalt and local tephrite through phonolite. These volcanics may represent the initiation of a third cycle of volcanism on Gran Canaria (Schmincke, 1982). As the Pliocene and Quaternary Cycles have the same $\mathrm{Sr}, \mathrm{Nd}$, and $\mathrm{Pb}$ isotopic compositions, they were probably derived from the same or similar source material (Hoernle et al., 1991) and thus will be considered together in this paper. The Pliocene-Quaternary volcanics, however, are isotopically distinct from the Miocene volcanics. The Pliocene-Quaternary volcanics make up $\sim 20 \%$ of the subaerial volume of Gran Canaria but compose $\ll 1 \%$ of the combined submarine and subaerial volume of the volcano (Schmincke, 1982; Hoernle, 1987).

\section{NOMENCLATURE}

Our classification of the Gran Canaria basalts, shown in Fig. 3, is similar to that of Le Bas et al. (1986a). The boundary between the alkali basalts and tholeiites is a best-fit line separating the basalts with normative hypersthene $\left[\mathrm{Fe}^{3+} /\left(\mathrm{Fe}^{3+}+\mathrm{Fe}^{2+}\right)=0 \cdot 2\right]$, picrites $(\mathrm{MgO} \geqslant 15)$ and tholeiites $(\mathrm{MgO}<15)$, from those with normative nepheline, alkali basalts. A few of the hypersthene-normative Pliocene basalts, however, fall within the alkali basalt field in Fig. 3. Division on the basis of $\mathrm{SiO}_{2}$ vs. the alkalis correlates better with other geochemical parameters than division based on the presence of hypersthene or nepheline in the norm. Although all of the Miocene picrites and tholeiites are hypersthene to slightly quartz normative, they have been classified as moderately alkaline or transitional in the past on the basis of their high alkali contents (Schmincke, 1976, 1982, 1990). These basalts fall on the boundary dividing Hawaiian tholeiitic from alkalic rocks (Macdonald \& Katsura, 1964) and thus are equivalent to Hawaiian transitional tholeiites. Alkali basalts and basanites are denoted by a single symbol to help reduce confusion by reducing the number of different symbols.

\section{PETROGRAPHY}

The following brief petrographic summary of the Gran Canaria basalts is based on the examination of more than 200 thin sections (for a more detailed discussion, see Schmincke, 1976, 1990; Hoernle, 1987). Miocene picrites and tholeiites have phenocrysts of olivine ( $<30 \%$, up to $50 \mathrm{~mm}$ ), titanaugite ( $<20 \%$, up to $50 \mathrm{~mm}$ ), and chromite $(\ll 1 \%$, $\ll 1 \mathrm{~mm}$ ). Olivine \pm clinopyroxene phenocrysts in the Miocene picrites commonly have partially resorbed edges and occur as glomerocrysts. Plagioclase $(<5 \%,<3 \mathrm{~mm})$ and $\mathrm{Fe}-\mathrm{Ti}$ oxides are present in samples with $\mathrm{MgO} \leqslant 6$. The groundmass consists of olivine, titanaugite, 
TABLE 1

Representative major $(X R F)$ and trace element $[X R F, I N A A$, and isotope dilution (ID)] data for Gran Canaria basalts listed in order of decreasing age

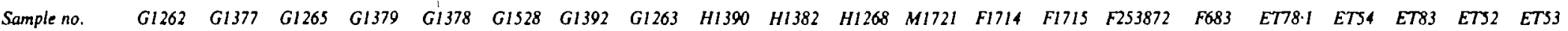

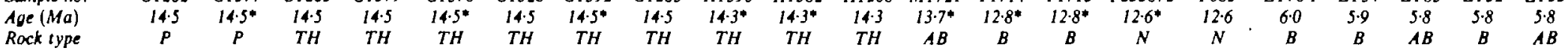

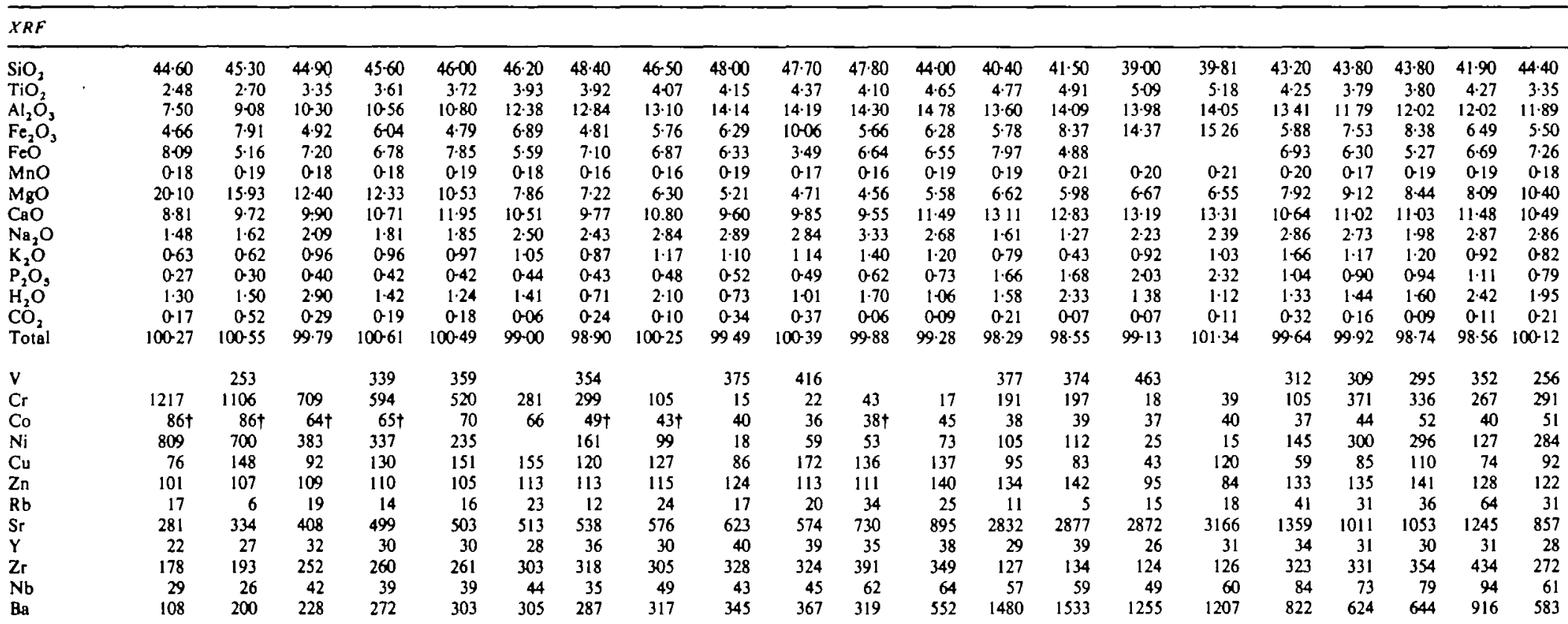




\begin{tabular}{|c|c|c|c|c|c|c|}
\hline$\tilde{n}_{i}^{\infty} \stackrel{\infty}{i}$ & 0 & 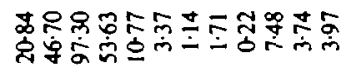 & $\sum_{\alpha} \bar{i} \div$ & & 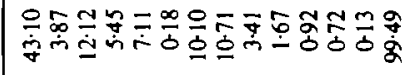 &  \\
\hline 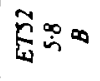 & & & $\frac{\pi}{\infty}=\infty$ & & 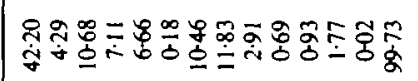 & 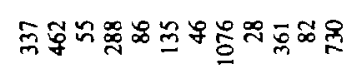 \\
\hline & & & $\frac{2}{2} \div \infty$ & & 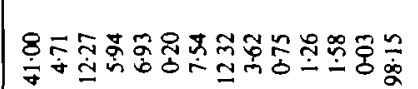 & 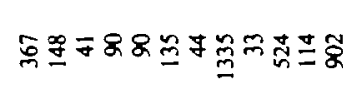 \\
\hline & 0 & 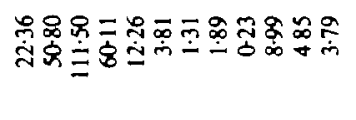 & $\sum_{\alpha}^{\infty} \div$ & & 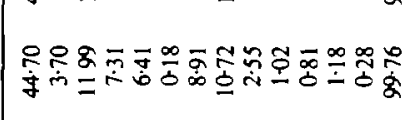 & 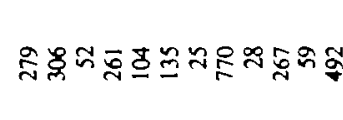 \\
\hline & & & & & 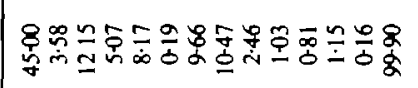 & 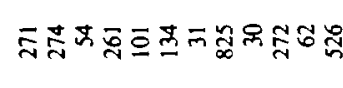 \\
\hline 8 & $\exists$ & 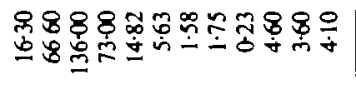 & & & 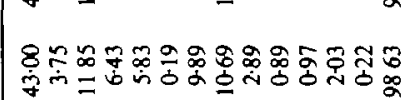 & 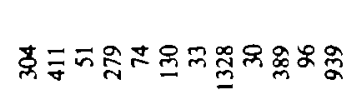 \\
\hline$\approx$ & $\exists$ & 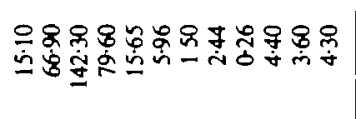 & $\sum_{\alpha}^{0}$ & &  & 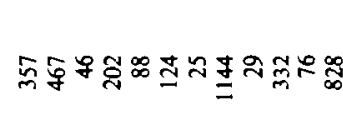 \\
\hline & & & 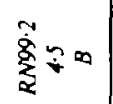 & & 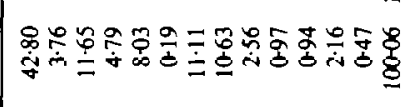 & 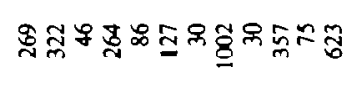 \\
\hline & & & $\div ₹$ & & 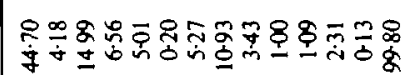 & 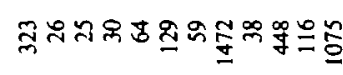 \\
\hline 2 & \pm & 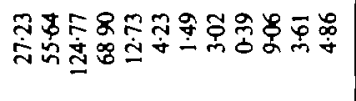 & $\sum+\infty$ & & 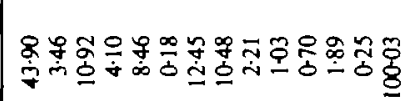 & 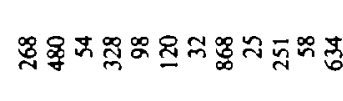 \\
\hline$m$ & $I$ & 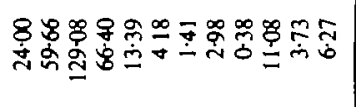 & & & 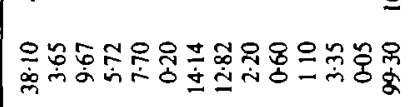 & 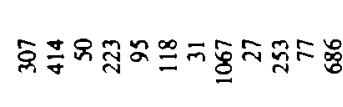 \\
\hline$\Sigma$ & 9 & 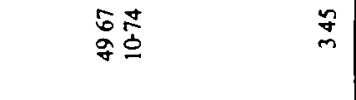 & $\mathbb{w}^{\infty}: i^{\circ}<$ & & 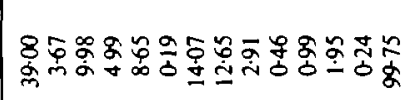 & 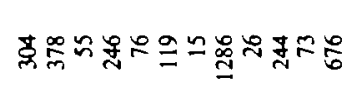 \\
\hline 25 & 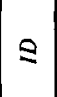 & 象高 & 总是之 & & 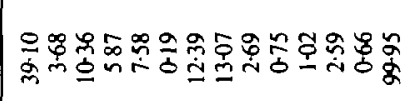 &  \\
\hline$\Sigma$ & $\exists$ & 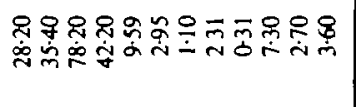 & & & 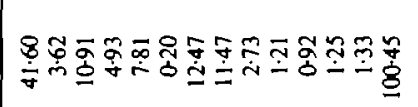 &  \\
\hline 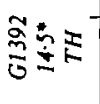 & 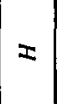 & 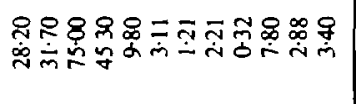 & $\hat{R}_{\omega}+\infty$ & & 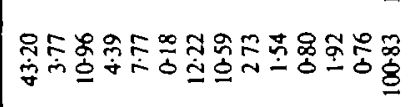 & 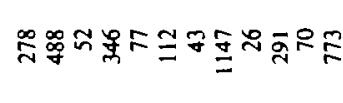 \\
\hline & & & & & 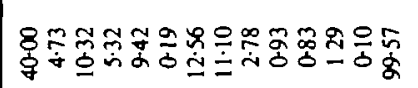 &  \\
\hline$\Sigma$ & $\geq$ & 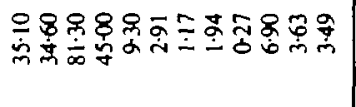 & 它的志 & & 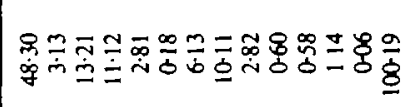 & 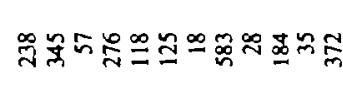 \\
\hline$\stackrel{2}{m}$ & $\approx$ & 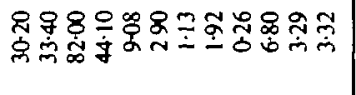 & 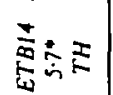 & & 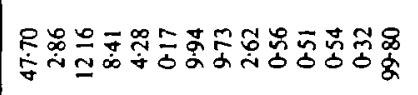 & 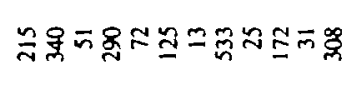 \\
\hline$I$ & $\geq$ & 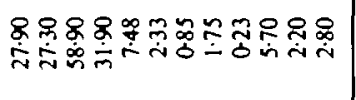 & हैं & & 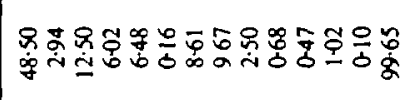 & 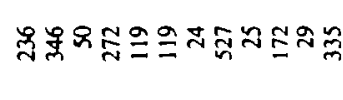 \\
\hline  & $=$ & 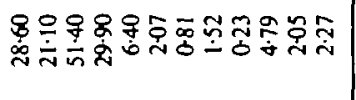 & $\tilde{\tilde{\tilde{c}}}= \pm$ & & 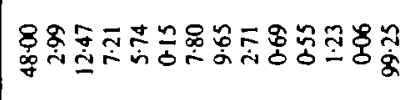 & 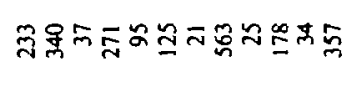 \\
\hline$\frac{1}{5}+2$ & \pm & 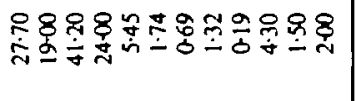 & $8= \pm$ & & 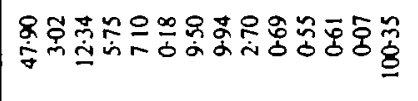 & 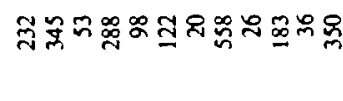 \\
\hline 告高高 & $\mp$ & 乌 30 & 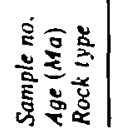 & $\left|\frac{4}{x}\right|$ & 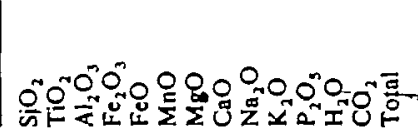 & 5 \\
\hline
\end{tabular}


TABLE 1 (continued)

\begin{tabular}{|c|c|c|c|c|c|c|c|c|c|c|c|c|c|c|c|c|c|c|c|c|c|}
\hline $\begin{array}{l}\text { Sample no. } \\
\text { Age (Ma) } \\
\text { Rock type }\end{array}$ & $\begin{array}{c}E T 60 \\
5 \cdot 7 \\
T H\end{array}$ & $\begin{array}{c}E T 62 \cdot 2 \\
5 \cdot 7 \\
T H\end{array}$ & $\begin{array}{c}E T 63 \cdot 3 \\
5 \cdot 7 \\
T H\end{array}$ & $\begin{array}{c}E T B 14 \\
5 \cdot 7^{*} \\
T H\end{array}$ & $\begin{array}{c}E T 63.1 \\
5.7 \\
T H\end{array}$ & $\begin{array}{c}E T 106 \\
5 \cdot 6^{*} \\
B\end{array}$ & $\begin{array}{c}E 795 \\
5 \cdot 4 \\
B\end{array}$ & $\begin{array}{c}E T 90 \\
5.4 \\
B\end{array}$ & $\begin{array}{c}\text { ETEA7 } \\
50 \\
N\end{array}$ & $\begin{array}{c}E T 88 \\
S 0^{*} \\
N\end{array}$ & $\begin{array}{c}E T B A 5 \\
S 0 \\
N\end{array}$ & $\begin{array}{c}R N 94 \\
48 \\
A B\end{array}$ & $\begin{array}{c}R N 70 \quad \\
4 \cdot 7 \\
A B\end{array}$ & $\begin{array}{c}R N Q 9.2 \\
4.5 \\
B\end{array}$ & $\begin{array}{c}R N 104 \\
44 \\
B\end{array}$ & $\begin{array}{c}R N 1249 \\
4 \cdot 4 \\
A B\end{array}$ & $\begin{array}{c}R N 86 \\
4 \cdot 3^{*} \\
A B\end{array}$ & $\begin{array}{c}R N 1257 \\
4 \cdot 2 \\
A B\end{array}$ & $\begin{array}{c}R N B 129 \\
4 \cdot 1 \\
B\end{array}$ & $\begin{array}{c}R N B \mid 25 \\
4 \cdot I \\
B\end{array}$ & $\begin{array}{c}R N Q S I \\
4 \cdot I^{*} \\
B\end{array}$ \\
\hline$I N A A$ & $c$ & & $I D$ & $w$ & $C$ & $c$ & C & & $w$ & $C$ & $w$ & $C$ & & $C$ & $C$ & $c$ & $c$ & C & & $H$ & $c$ \\
\hline $\begin{array}{l}\text { Sc } \\
\text { La } \\
\mathrm{Ce} \\
\mathrm{Nd} \\
\mathrm{Sm} \\
\mathrm{Eu} \\
\mathrm{Tb} \\
\mathrm{Yb} \\
\mathrm{Lu} \\
\mathrm{Hf} \\
\mathrm{Ta} \\
\mathrm{Th}\end{array}$ & $\begin{array}{r}23 \cdot 23 \\
27 \cdot 35 \\
63 \cdot 00 \\
36-97 \\
8.33 \\
2.69 \\
101 \\
1.73 \\
0.22 \\
5.29 \\
2.39 \\
2.21\end{array}$ & & $\begin{array}{r}31.07 \\
7.48\end{array}$ & $\begin{array}{r}22.60 \\
23.80 \\
50.20 \\
29.40 \\
7.56 \\
2.59 \\
0.94 \\
1.77 \\
0.24 \\
5.00 \\
2.00 \\
2.10\end{array}$ & $\begin{array}{r}23.80 \\
26.66 \\
59-05 \\
35.63 \\
8.15 \\
2.67 \\
0.97 \\
1.70 \\
0.22 \\
5.54 \\
2.17 \\
2.24\end{array}$ & $\begin{array}{r}25.86 \\
44.80 \\
9820 \\
54.46 \\
11.48 \\
3.31 \\
1.08 \\
1.53 \\
0.18 \\
8.13 \\
4.61 \\
3.78\end{array}$ & $\begin{array}{r}23 \cdot 26 \\
59.00 \\
119 \cdot 20 \\
57.63 \\
11 \cdot 43 \\
3.25 \\
1 \cdot 13 \\
1.80 \\
0.22 \\
7.86 \\
4.55 \\
5.44\end{array}$ & & $\begin{array}{r}2430 \\
51.50 \\
95 \cdot 60 \\
47.10 \\
10.68 \\
3.41 \\
1.08 \\
1.91 \\
0.21 \\
5.80 \\
4.00 \\
5.60\end{array}$ & $\begin{array}{r}23 \cdot 57 \\
5350 \\
110-00 \\
59-18 \\
1160 \\
3.45 \\
1.14 \\
1.53 \\
0.17 \\
6.80 \\
4.26 \\
5.24\end{array}$ & $\begin{array}{r}24 \cdot 80 \\
59 \cdot 10 \\
112.20 \\
55 \cdot 10 \\
12.42 \\
3.87 \\
1.26 \\
2.14 \\
0.21 \\
6.50 \\
4.40 \\
5.80\end{array}$ & $\begin{array}{r}22 \cdot 36 \\
40.20 \\
86 \cdot 10 \\
46 \cdot 02 \\
9 \cdot 23 \\
2.90 \\
0.96 \\
1.58 \\
0.19 \\
6 \cdot 71 \\
3.93 \\
3.87\end{array}$ & & $\begin{array}{r}21 \cdot 32 \\
51 \cdot 70 \\
116.30 \\
60.71 \\
12.41 \\
393 \\
1.31 \\
1.81 \\
0.22 \\
9.76 \\
511 \\
4.27\end{array}$ & $\begin{array}{r}25 \cdot 44 \\
62 \cdot 10 \\
132 \cdot 60 \\
68 \cdot 11 \\
12.82 \\
3.88 \\
1.29 \\
1 \cdot 72 \\
0.22 \\
9.44 \\
4.83 \\
5 \cdot 10\end{array}$ & $\begin{array}{r}20.20 \\
76 \cdot 30 \\
158 \cdot 10 \\
73 \cdot 23 \\
13.53 \\
4 \cdot 15 \\
1.32 \\
1.97 \\
0.25 \\
10-24 \\
6.23 \\
7.02\end{array}$ & $\begin{array}{r}20.79 \\
4470 \\
96.70 \\
54.63 \\
11.50 \\
3.45 \\
1.20 \\
1.84 \\
0.23 \\
7.55 \\
40.8 \\
3.57\end{array}$ & $\begin{array}{r}2396 \\
39.99 \\
97.29 \\
44.10 \\
10-41 \\
3.65 \\
1.28 \\
2.08 \\
0.25 \\
7.72 \\
3.79 \\
2.96\end{array}$ & & $\begin{array}{r}25.50 \\
60.60 \\
135-00 \\
68.00 \\
13.00 \\
4.08 \\
1.43 \\
1.68 \\
0.22 \\
9.20 \\
6.40 \\
5.90\end{array}$ & $\begin{array}{r}20-99 \\
70-60 \\
145.50 \\
69 \cdot 28 \\
14.00 \\
4.10 \\
1.41 \\
1.84 \\
0.22 \\
9.47 \\
5.60 \\
6.16\end{array}$ \\
\hline $\begin{array}{l}\text { Sample no. } \\
\text { Age (Ma) } \\
\text { Rock iype }\end{array}$ & $\begin{array}{c}R N 677 \\
1 \cdot 1^{\circ} \\
B\end{array}$ & $\begin{array}{c}R N 1258 \\
4 \cdot 1 \\
B\end{array}$ & $\begin{array}{c}R N 1130 \\
3 \cdot 8 \\
B\end{array}$ & $\begin{array}{c}R N C 49 \\
3.7 \\
B\end{array}$ & $\begin{array}{c}R N B 105 \\
36 \\
B\end{array}$ & $\begin{array}{c}L L 1424 \\
3 \cdot 1^{*} \\
N\end{array}$ & $\begin{array}{c}L L 111 S \\
30 \\
N\end{array}$ & $\begin{array}{c}L L C 16 \\
2 \cdot 9 \\
N\end{array}$ & $\begin{array}{c}L L 1138 \\
2 \cdot 8 \\
B\end{array}$ & $\begin{array}{c}L L 1114 I \\
2 \cdot 6^{*} \\
N\end{array}$ & $\begin{array}{c}L L 1142 \\
2 \cdot 4^{*} \\
N\end{array}$ & $\begin{array}{c}\text { LLC9 } \\
1.9 \\
N\end{array}$ & $\begin{array}{c}L L 1415 \\
18^{*} \\
N\end{array}$ & $\begin{array}{c}5 L C 22 \\
1 \cdot 8 \\
N\end{array}$ & $\begin{array}{c}Q C 21 \\
1 \cdot 3 \\
N\end{array}$ & $\begin{array}{c}Q 307 \\
1-0^{*} \\
N\end{array}$ & $\begin{array}{l}Q D 6 \\
0-3 \\
\mathrm{AB}\end{array}$ & $\begin{array}{c}Q 1414 \\
02^{*} \\
B\end{array}$ & $\begin{array}{c}Q 1369 \\
0-0 \\
B\end{array}$ & $\begin{array}{c}Q 1499 \\
0-0 \\
B\end{array}$ & $\begin{array}{c}Q 1433 \\
0-0 \\
B\end{array}$ \\
\hline \multicolumn{22}{|l|}{$X R F$} \\
\hline $\begin{array}{l}\mathrm{SiO}_{2} \\
\mathrm{TiO}_{2} \\
\mathrm{Al}_{2} \mathrm{O}_{3} \\
\mathrm{Fe}_{2} \mathrm{O}_{3} \\
\mathrm{FeO}_{3} \\
\mathrm{MnO} \\
\mathrm{M}_{8} \mathrm{O} \\
\mathrm{CaO} \\
\mathrm{Na}_{2} \mathrm{O} \\
\mathrm{K}_{2} \mathrm{O} \\
\mathrm{P}_{2} \mathrm{O}_{3} \\
\mathrm{H}_{2} \mathrm{O} \\
\mathrm{CO}_{2} \\
\text { Total }\end{array}$ & $\begin{array}{r}42.00 \\
4.27 \\
11.90 \\
8.87 \\
4.56 \\
0.19 \\
10-21 \\
11.11 \\
3.25 \\
0-98 \\
1.14 \\
1.36 \\
0.12 \\
99-96\end{array}$ & $\begin{array}{r}43 \cdot 70 \\
3.63 \\
12.24 \\
6.39 \\
6.19 \\
0.19 \\
10-04 \\
11 \cdot 00 \\
2.98 \\
1.11 \\
0.89 \\
2.26 \\
0.11 \\
100-73\end{array}$ & $\begin{array}{r}41 \cdot 40 \\
3.68 \\
10-55 \\
6.37 \\
7.40 \\
0.19 \\
12.24 \\
11.84 \\
2.55 \\
0-65 \\
0.88 \\
1.90 \\
0.20 \\
99-85\end{array}$ & $\begin{array}{r}40-50 \\
3.88 \\
10.31 \\
4.87 \\
7.73 \\
0.18 \\
12.70 \\
11.63 \\
2.94 \\
1.47 \\
0.81 \\
1.35 \\
0.05 \\
98.42\end{array}$ & $\begin{array}{r}43-60 \\
3.60 \\
11.76 \\
5.66 \\
7.58 \\
0.19 \\
10-15 \\
11.52 \\
2.31 \\
0.88 \\
0.78 \\
1.96 \\
0-03 \\
100-02\end{array}$ & $\begin{array}{r}38 \cdot 60 \\
3.98 \\
9.22 \\
9.93 \\
4.02 \\
0.20 \\
14.02 \\
12.88 \\
2.72 \\
1.18 \\
1.45 \\
0.92 \\
0.38 \\
99.50\end{array}$ & $\begin{array}{r}40-60 \\
3.42 \\
10.76 \\
5.80 \\
7.30 \\
0.20 \\
13.30 \\
12.40 \\
2.20 \\
0.60 \\
133 \\
1.50 \\
0.19 \\
99-60\end{array}$ & $\begin{array}{r}39.20 \\
3.52 \\
9.84 \\
7.61 \\
5.17 \\
0.19 \\
14.07 \\
12.55 \\
2.68 \\
1.24 \\
0.98 \\
1.18 \\
0-05 \\
98.28\end{array}$ & $\begin{array}{r}41.00 \\
3.64 \\
9.97 \\
8.14 \\
4.75 \\
0.18 \\
1393 \\
11.89 \\
2.66 \\
0-58 \\
0.86 \\
1.89 \\
0.15 \\
99-64\end{array}$ & $\begin{array}{r}38 \cdot 60 \\
3 \cdot 51 \\
9 \cdot 78 \\
8 \cdot 61 \\
4 \cdot 61 \\
0.20 \\
14 \cdot 28 \\
13 \cdot 29 \\
2 \cdot 33 \\
103 \\
1.46 \\
1 \cdot 13 \\
0.28 \\
99.11\end{array}$ & $\begin{array}{r}38.70 \\
4.01 \\
10.15 \\
9.72 \\
4.51 \\
0.22 \\
1321 \\
12.91 \\
268 \\
1.11 \\
141 \\
1.61 \\
0.21 \\
100-45\end{array}$ & $\begin{array}{r}36 \cdot 90 \\
4.09 \\
9-98 \\
5.57 \\
8 \cdot 11 \\
0.21 \\
13.35 \\
13.16 \\
2.70 \\
0-62 \\
1.73 \\
1.71 \\
0-03 \\
98.16\end{array}$ & $\begin{array}{r}37 \cdot 40 \\
3.78 \\
9 \cdot 35 \\
5.71 \\
6.69 \\
0.20 \\
14.02 \\
14.27 \\
2.97 \\
1.41 \\
1.38 \\
0.56 \\
138 \\
99.12\end{array}$ & $\begin{array}{r}37.00 \\
3.20 \\
8.78 \\
4.71 \\
7.50 \\
0.20 \\
16.77 \\
13.85 \\
2.79 \\
0.84 \\
1.38 \\
1.13 \\
0-06 \\
98.21\end{array}$ & $\begin{array}{r}38 \cdot 70 \\
3.39 \\
10-29 \\
4.92 \\
7 \cdot 14 \\
0.19 \\
1320 \\
13.68 \\
3.25 \\
1.06 \\
131 \\
0.85 \\
0.03 \\
98.01\end{array}$ & $\begin{array}{r}38.40 \\
5.62 \\
9.76 \\
412 \\
10.33 \\
0.19 \\
12.68 \\
11.56 \\
2.90 \\
1.15 \\
1.17 \\
1.34 \\
0.05 \\
99.27\end{array}$ & $\begin{array}{r}0-18 \\
9-04 \\
10-88 \\
3-58 \\
114 \\
0-94 \\
0-47 \\
013 \\
100-02\end{array}$ & $\begin{array}{r}42 \cdot 40 \\
4 \cdot 20 \\
12 \cdot 30 \\
406 \\
8 \cdot 40 \\
0 \cdot 19 \\
10 \cdot 36 \\
12 \cdot 11 \\
3.06 \\
1 \cdot 51 \\
0-96 \\
0-19 \\
0-05 \\
99-79\end{array}$ & $\begin{array}{r}41 \cdot 70 \\
3.35 \\
10.50 \\
3.15 \\
8.80 \\
0.18 \\
16.20 \\
11.20 \\
2.47 \\
1.33 \\
0.67 \\
0.10 \\
0.05 \\
99.70\end{array}$ & $\begin{array}{r}41 \cdot 90 \\
3.70 \\
11.60 \\
3.51 \\
8.10 \\
0.18 \\
12.40 \\
1210 \\
2.63 \\
1.59 \\
0.78 \\
0-60 \\
0-06 \\
99.15\end{array}$ & $\begin{array}{r}43.20 \\
3.80 \\
11.90 \\
3.96 \\
8.52 \\
0.18 \\
11.20 \\
11.40 \\
3.13 \\
1.50 \\
080 \\
0.20 \\
0.15 \\
9994\end{array}$ \\
\hline
\end{tabular}




\begin{tabular}{|c|c|c|c|c|c|c|c|c|c|c|c|c|c|c|c|c|c|c|c|c|c|}
\hline $\begin{array}{l}\text { Sample no. } \\
\text { Age (Ma) } \\
\text { Rock rype }\end{array}$ & $\begin{array}{c}R N 677 \\
4 \cdot 1^{*} \\
B\end{array}$ & $\begin{array}{c}R N 1258 \\
4 \cdot 1 \\
B\end{array}$ & $\begin{array}{c}R N 1130 \\
3.8 \\
B\end{array}$ & $\begin{array}{c}R N C 49 \\
3 \cdot 7 \\
B\end{array}$ & $\begin{array}{c}R N B 105 \\
3 \cdot 6 \\
B\end{array}$ & $\begin{array}{c}L L 1424 \\
3 \cdot 1^{*} \\
N\end{array}$ & $\begin{array}{c}\text { LL111S } \\
3-0 \\
N\end{array}$ & $\begin{array}{c}L L C 16 \\
2-9 \\
N\end{array}$ & $\begin{array}{c}L L 1138 \\
2 \cdot 8 \\
B\end{array}$ & $\begin{array}{c}L L J 1141 \\
26^{*} \\
N\end{array}$ & $\begin{array}{c}L L 1142 \\
2.4^{*} \\
N\end{array}$ & $\begin{array}{c}\angle L C 9 \\
1.9 \\
N\end{array}$ & $\begin{array}{c}L L 1415 \\
1.8^{*} \\
N\end{array}$ & $\begin{array}{c}L L C 22 \\
1 \cdot 8 \\
N\end{array}$ & $\begin{array}{c}Q C 21 \\
1 \cdot 3 \\
N\end{array}$ & $\begin{array}{c}Q 307 \\
1.0^{*} \\
N\end{array}$ & $\begin{array}{l}Q D 6 \\
0-3 \\
\mathrm{AB}\end{array}$ & $\begin{array}{c}Q 1414 \\
0.2 * \\
B\end{array}$ & $\begin{array}{c}Q 1369 \\
00 \\
B\end{array}$ & $\begin{array}{c}Q 1499 \\
0-0 \\
B\end{array}$ & $\begin{array}{c}Q / 433 \\
0-0 \\
B\end{array}$ \\
\hline \multicolumn{22}{|l|}{$X R F$} \\
\hline$v$ & 339 & 300 & 299 & 280 & 294 & 274 & 300 & 276 & 315 & 269 & 328 & 290 & 296 & 265 & 282 & & 342 & 360 & 293 & & 282 \\
\hline $\mathrm{Cr}$ & 292 & 352 & 364 & 424 & 324 & 481 & 510 & 462 & 719 & 445 & 354 & 408 & 373 & 592 & 410 & 358 & 337 & 438 & 808 & 744 & 385 \\
\hline Co & 54 & 50 & 57 & 58 & 54 & 79 & 73 & 61 & 82 & 77 & 77 & 52 & 68 & 63 & 57 & & 65 & 59 & 69 & 59 & 60 \\
\hline $\mathrm{Ni}$ & 230 & 221 & 290 & 232 & 254 & 338 & 144 & 331 & 377 & 322 & 288 & 223 & 337 & 446 & 322 & 133 & 227 & 152 & 424 & 245 & 229 \\
\hline $\mathrm{Cu}$ & 86 & 65 & 95 & 69 & 96 & 88 & 62 & 81 & 90 & 83 & 91 & 69 & 102 & 87 & 100 & 63 & 68 & 70 & 68 & 87 & 62 \\
\hline $\mathrm{Zn}$ & 128 & 123 & 128 & 111 & 136 & 129 & 107 & 120 & 109 & 118 & 139 & 121 & 116 & 105 & 113 & 116 & 122 & 108 & 88 & 100 & 100 \\
\hline $\overrightarrow{\mathbf{R b}}$ & 24 & 34 & 29 & 24 & 48 & 18 & 64 & 30 & 75 & 18 & 41 & 12 & 25 & 14 & 35 & 15 & 21 & 31 & 21 & 30 & 37 \\
\hline Sr & 1234 & 1061 & 858 & 1358 & 911 & 1392 & 1356 & 1204 & 978 & 1502 & 1490 & 1787 & 1355 & 1333 & 1439 & 1187 & 1198 & 1109 & 830 & 974 & 1038 \\
\hline$Y$ & 30 & 29 & 27 & 28 & 27 & 37 & 36 & 33 & 16 & 38 & 39 & 39 & 37 & 33 & 32 & 28 & 33 & 33 & 30 & 33 & 24 \\
\hline $\mathrm{Zr}_{\mathrm{r}}$ & 317 & 342 & 252 & 255 & 275 & 358 & 321 & 303 & 292 & 262 & 351 & 264 & 317 & 260 & 289 & 329 & 344 & 276 & 212 & 279 & 279 \\
\hline $\mathrm{Nb}$ & 80 & 85 & 64 & 66 & 66 & 87 & 80 & 86 & 77 & 79 & 91 & 88 & 90 & 100 & 94 & 73 & 83 & 64 & 57 & 67 & 62 \\
\hline Ba & 838 & 809 & 620 & 755 & 639 & 1156 & 745 & 751 & 710 & 703 & 660 & 882 & 759 & 1005 & 941 & 603 & 698 & 696 & 509 & 594 & 435 \\
\hline INAA & $C$ & C & $C$ & $H$ & $H$ & $H$ & & $H$ & & $H$ & $w$ & $H$ & $H$ & $H$ & $W$ & $W$ & $X R F$ & $H$ & $w$ & $w$ & $w$ \\
\hline Sc & 20005 & 21.69 & $24 \cdot 50$ & $23 \cdot 50$ & $24 \cdot 60$ & $23 \cdot 60$ & & $24 \cdot 10$ & & $24 \cdot 00$ & $24 \cdot 40$ & $26 \cdot 30$ & $23 \cdot 70$ & $24 \cdot 10$ & 2360 & $23 \cdot 20$ & $17-00$ & $28 \cdot 50$ & 29.80 & 3100 & 28.50 \\
\hline La & $63 \cdot 60$ & $65 \cdot 10$ & 48.80 & 56.60 & $50-10$ & 84.60 & & 71.90 & & $103-00$ & 82.50 & 97.00 & 101.00 & 9600 & 92.90 & $60-40$ & $94-\infty$ & $59-20$ & $47 \cdot 10$ & 5300 & $49-20$ \\
\hline $\mathrm{Ce}$ & $131 \cdot 50$ & $129-80$ & 10380 & $120-00$ & $106-00$ & $186-00$ & & 15600 & & 217.00 & 169.80 & 218.00 & 21600 & 197.00 & 186.00 & 129.70 & $91-00$ & 13100 & $85 \cdot 40$ & 103.50 & 104.50 \\
\hline Nd & 70.19 & 63.74 & $60-67$ & $59-00$ & 5340 & $92-00$ & & $73-00$ & & $101-\infty 0$ & $84 \cdot 40$ & 10600 & $100-00$ & $88-00$ & $84 \cdot 30$ & 62.80 & $68-00$ & $64-00$ & $41-00$ & $50-20$ & 51.70 \\
\hline $\mathrm{Sm}$ & $13 \cdot 18$ & 11.87 & 11.28 & 11.40 & $10-50$ & 1690 & & $13 \cdot 60$ & & 17.60 & 16.60 & 1880 & 1750 & 15.50 & 14.45 & 12.83 & 1400 & 12.00 & $9-01$ & $10-33$ & $10-13$ \\
\hline Eu & 404 & 3.52 & $3 \cdot 46$ & 3.57 & $3 \cdot 36$ & 521 & & $4 \cdot 12$ & & 5.34 & 5.15 & 5.78 & $5 \cdot 26$ & 4.63 & 443 & $4 \cdot 15$ & & 368 & 2.79 & $3 \cdot 19$ & 3.28 \\
\hline $\mathrm{Tb}$ & 1.33 & $1 \cdot 18$ & 1.18 & 1.20 & 1.22 & $1-62$ & & 1.38 & & 1.67 & 1.59 & 1.78 & 1.77 & 1.47 & 1.43 & 1.33 & & 1.23 & 0.89 & 1.05 & 106 \\
\hline $\mathrm{Yb}$ & 1.91 & 1.79 & 1.63 & 1.54 & 1.69 & 1.75 & & 1.75 & & 1.87 & 268 & $2 \cdot 15$ & 1.88 & 170 & $2 \cdot 10$ & 1.85 & & 1.86 & 1.74 & $2 \cdot 13$ & $2 \cdot 14$ \\
\hline Lu & $0-24$ & 0.23 & 0.20 & $0-22$ & 023 & $0-23$ & & 0.24 & & $0-27$ & $0-24$ & $0-29$ & $0-25$ & $0-23$ & 0.28 & $0-26$ & & 0.26 & 0.22 & 0.24 & 0.26 \\
\hline $\mathrm{Hf}$ & 8.58 & 8.91 & 6.94 & 6.80 & $6-80$ & 9.20 & & 7.90 & & 6.80 & 9.10 & 7.00 & 7.80 & 6.60 & 7.60 & $8-00$ & & $6-90$ & $5 \cdot 20$ & $6 \cdot 70$ & 660 \\
\hline $\mathrm{Ta}$ & 5.10 & $5-08$ & 3.94 & $5 \cdot 20$ & 4.73 & $7 \cdot 30$ & & 650 & & $6 \cdot 30$ & & $6-40$ & $7 \cdot 10$ & 6.70 & 5.40 & 4.60 & & 500 & $3 \cdot 40$ & 3.90 & 3.80 \\
\hline $\mathrm{Th}$ & $5-67$ & 6.58 & 4.22 & 5.90 & $5 \cdot 10$ & $8 \cdot 30$ & & 7.90 & & $10-30$ & 7.90 & 9.50 & $10-60$ & $11 \cdot 10$ & 9.50 & $5 \cdot 10$ & & $6-00$ & $4 \cdot 40$ & $5 \cdot 30$ & 5.30 \\
\hline
\end{tabular}

The letters preceding the sample number correspond to the following formation names: G-Guigui, H-Hogarzales, M-Mogan, F-Fataga, ET-El Tablero, RN-Roque Nublo, LL-Los Listos and Los Pechos, Q-Quaternary. Samples M1721, F253872, and F683 are from basalt units T4, T5, and T6, respectively. About 120 volcanic units have been dated on Gran Canaria.

*Ages from dated units [Lietz \& Schmincke, 1975; McDougall \& Schmincke, 1976; Feraud et al., 1981; Schmincke et al., in prep.; H -U. Schmincke, unpub. data; ages from studies predating 1979 were multiplied by 10266 (Mankinen \& Dalrymple, 1979)]. The other ages are estimated based on stratigraphic position and paleomagnetic data (H -U. Schmincke \& U. Bleil, unpub. data). Based on detailed Ar/Ar dating of the Mogan ignimbrites (van den Bogaard et al., 1988), the ages for the Guigui and Hogarzales basalts from McDougall \& Schmiacke (1976) are too young and have been mod, fied slightly. Some of ages reported by Hoernle et al. (1991) for the same samples in this study have been changed as a result of new age data (Schmıncke et al., in prep.; H.-U. Schmincke, unpub. data). Errors in the absolute ages of the undated flows are estimated at $\pm 0-25 \mathrm{Ma}$ at the $1 \sigma$ confidence level.

The abbreviations for rock type are P-picrite, TH-tholeiite, AB-alkali basalt, B-basanite, N-nephelinite, MN—melilite nephelinite. Some of the XRF analyses are from Schmincke (1976, 1982, 1987, and unpub. data) and Hoernle (1987). The major element and some of the trace element ( $\mathrm{Ni}, \mathrm{Cu}, \mathrm{Sr}, \mathrm{Y}$, and $\mathrm{Nb}$ ) data for F683 are from XRF (Cousens et al., 1990 ), the other trace element values are from INAA (this study).

The values from INAA. analyses are from ID (Hoernle et al., 1991). The Sr, Nd, and Pb isotope ratios and the ID data (Rb, Sr, Sm, Nd, U. Th, and Pb) have been reported by Hoernle el al. (1991; most of the samples in this table) and Cousens et al. (1990; sample F682, same flow as F683, and unit T4). When available, the ID data were used in preference to XRF or INAA data in plots in this paper and in Hoernte \& Schmincke (this volume). 


\section{GRAN CANARIG MAFIC UOLCANICS}

Miocene (Filled Symbols); Pliocene-Quaternary (Open Symbols)
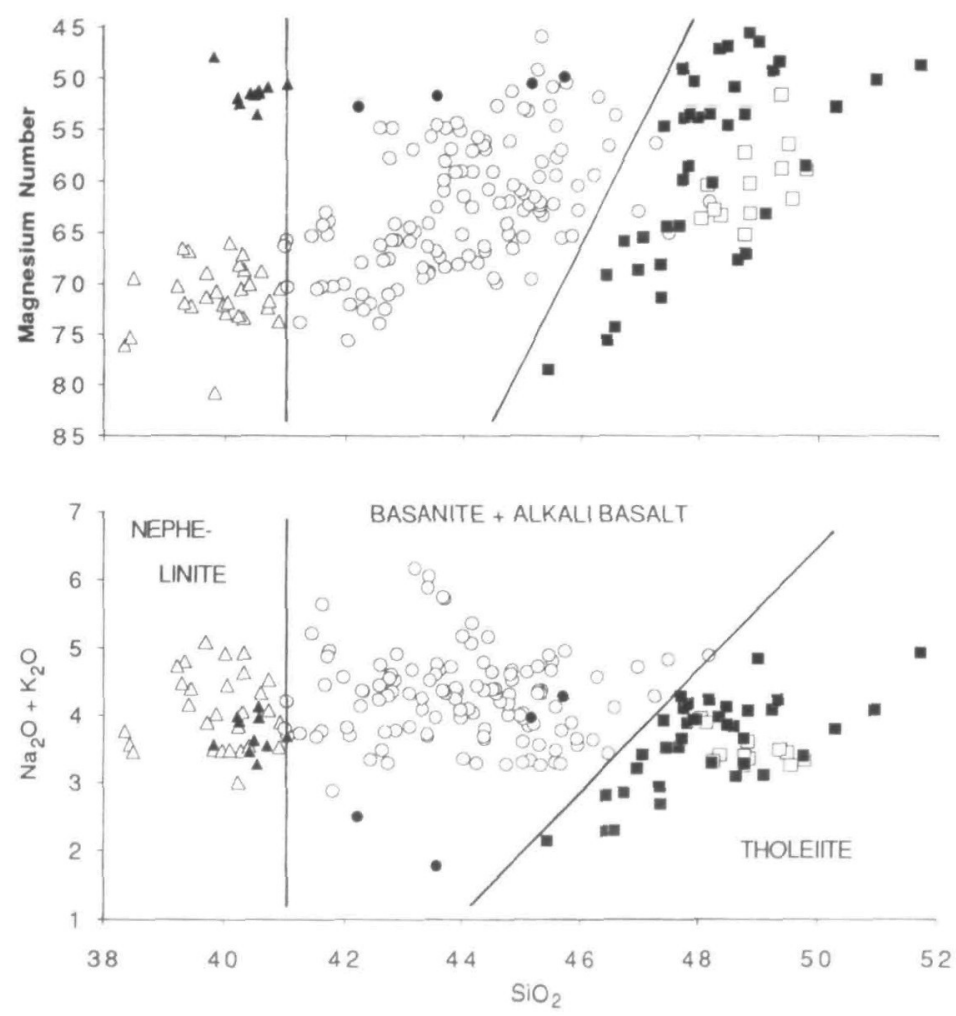

FiG. 3. $\mathrm{SiO}_{2}$ vs. the alkalis $\left(\mathrm{Na}_{2} \mathrm{O}+\mathrm{K}_{2} \mathrm{O}\right)$ and magnesium number with $\left(\mathrm{Fe}^{3+} /\left(\mathrm{Fe}^{3+}+\mathrm{Fe}^{2+}\right)=0-2\right.$ for $\mathrm{Gran}$ Canaria mafic volcanics. The boundaries between nephelinites (triangles), basanites + alkali basalts (circles), and picrites +tholeiites (squares) are shown on both diagrams. The boundary between tholeiites and alkali basalts roughly divides the samples with normative hypersthene or quartz from those with normative nepheline. Boundary between nephelinites and basanites is from Le Bas et al. (1986b).

plagioclase, and $\mathrm{Fe}-\mathrm{Ti}$ oxides. The Miocene alkali basalts, basanites, and nephelinites are clinopyroxene $(<7 \%)$ and olivine $(<3 \%)$ phyric. The groundmass is made up of olivine, titanaugite, plagioclase, Fe-Ti oxides, and apatite (Schmincke, 1987).

The Pliocene tholeiites contain olivine $(<11 \%,<3 \mathrm{~mm})$ with chromite inclusions $(\ll 1 \%$, $\ll 1 \mathrm{~mm})$, plagioclase $(<7 \%,<3 \mathrm{~mm})$, and Fe-Ti oxide $(<2 \%,<1 \mathrm{~mm})$ phenocrysts. The rare titanaugite phenocrysts are in most cases broken and/or partially resorbed. The groundmass consists of olivine, plagioclase, titanaugite, and Fe-Ti oxides.

The Pliocene and Quaternary alkali basalts and basanites contain phenocrysts of olivine $(<15 \%,<5 \mathrm{~mm})$, titanaugite $(<25 \%,<6 \mathrm{~mm})$, Fe-Ti oxides $(<8 \%,<2 \mathrm{~mm})$, and chromite $(\ll 1 \%, \ll 1 \mathrm{~mm})$. As $\mathrm{MgO}$ decreases, the abundance of olivine phenocrysts decreases, whereas the abundance of titanaugite and Fe-Ti oxides increases (Hoernle, 1987). Plagioclase, amphibole, and apatite are present in some rocks with $\mathrm{MgO}<6$. The groundmass consists of olivine, plagioclase, titanaugite, Fe-Ti oxides, amphibole, apatite, 
and glass. The basanites also contain groundmass nepheline, and some of the primitive basanites also contain primary(?) carbonate.

The Pliocene and Quaternary nephelinites are aphyric to moderately phyric. Olivine $(\leqslant 15 \%,<5 \mathrm{~mm})$ is the major phenocryst phase and minor titanaugite is present in some rocks. Olivine, titanaugite, oxides, nepheline, apatite, and primary(?) carbonate form the groundmass. The melilite-bearing nephelinites also contain perovskite and groundmass sodalite, as well as spinel lherzolite xenoliths and abundant olivine, enstatite, and chrome spinel xenocrysts. Quaternary(?) nephelinite dikes in the cliff above Risco Blanco at the head of Barranco de Tirajana and at Cazadores also contain spinel lherzolite xenoliths.

\section{ANALYTICAL METHODS AND SAMPLE SELECTION}

Approximately 200 basalts from Gran Canaria were analyzed for major and trace elements (Table 1) by X-ray fluorescence on fused discs using a Phillips PW1400 Spectrometer at the Ruhr Universität in Bochum, Germany. $\mathrm{FeO}, \mathrm{H}_{2} \mathrm{O}^{+}, \mathrm{H}_{2} \mathrm{O}^{-}$, and $\mathrm{CO}_{2}$ were determined using wet chemical techniques. Analysts were Henni Niephaus, Beate Schulz, and Kaj Hoernle. The procedures used have been described in more detail by Flower et al. (1983) and Feraud et al. (1981). The data in Table 1 are listed as analyzed. The data in plots and the data used for the CIPW norms are recalculated on a volatile-free basis with $\mathrm{Fe}^{3+} /\left(\mathrm{Fe}^{3+}+\right.$ $\left.\mathrm{Fe}^{2+}\right)=0 \cdot 2$.

Approximately 70 samples were also analyzed for $\mathrm{Sc}, \mathrm{Cr}, \mathrm{Co}, \mathrm{La}, \mathrm{Ce}, \mathrm{Nd}, \mathrm{Sm}, \mathrm{Eu}, \mathrm{Tb}, \mathrm{Yb}$, Lu, Hf, Ta, and Th (Table 1) by INAA at Washington University in St. Louis, Missouri (analyst Pat Castillo), at the University of Leuven (analyst Jan Hertogen), and at Oregon State University Radiation Center (analyst Bob Walker). Average values are reported for samples which were multiply analyzed. Samples from the same formation or basalt group were sent to at least two laboratories to minimize the effects of inter-laboratory bias. In addition, comparisons of the standard and sample data from the three laboratories showed no significant inter-laboratory bias for the elements reported except that Ta values from the University of Leuven appear to be systematically too high when compared with Ta from other laboratories. More detailed descriptions of the INAA method and error analyses for the various laboratories have been given by Jacobs et al. (1977) and Lindstrom \& Korotev (1983) (Washington University), Hertogen \& Gijbels (1971) (University of Leuven), and Laul \& Schmitt (1973) (Oregon State University). A detailed comparison of analytical methods will be provided in a forthcoming paper on Holocene volcanics from the Canary Islands.

Additional sources of major and trace element data used in some of the plots are Schmincke (1976, 1982, 1990, unpub. data), Crisp (1984), Hoernle (1987, unpub. data), Cousens (1990), Cousens et al. (1990), H. R. Bowman \& H.-U. Schmincke (unpub. data), and H.-U. Schmincke \& P. T. Robinson (unpub. data). $\mathrm{Sr}-\mathrm{Nd}-\mathrm{Pb}$ isotopic compositions and $\mathrm{Rb}, \mathrm{Sr}, \mathrm{Sm}, \mathrm{Nd}, \mathrm{Pb}, \mathrm{U}$, and $\mathrm{Th}$ concentrations determined by isotope dilution have been reported for 66 of these samples by Hoernle et al. (1991).

Although Gran Canaria contains a wide variety of evolved volcanics including hawaiite through trachyte and peralkaline rhyolite and tephrite through phonolite, only mafic volcanic samples [picrite, tholeiite, alkali basalt, basanite, and nephelinite, using the Le Bas et al. (1986a) classification] are included in this study. Several criteria were used to screen data for alteration. Samples which contained $>2.5$ wt. $\% \mathrm{H}_{2} \mathrm{O}$ and $>0.5$ wt. $\% \mathrm{CO}_{2}$, and samples that showed significant groundmass alteration in thin section, especially those that contained secondary micas, were excluded. As a result of high F contents (as high as $2 \mathrm{wt} . \%$ ), many totals, without $\mathrm{F}$, summed to less than $99 \%$. 


\section{MAJOR AND TRACE ELEMENT DATA}

The $m g$-numbers $\left[=\mathrm{Mg} /\left(\mathrm{Mg}+\mathrm{Fe}^{2+}\right)\right.$ with $\left.\mathrm{Fe}^{3+} /\left(\mathrm{Fe}^{3+}+\mathrm{Fe}^{2+}\right)=0-2\right]$ for the Gran Canaria mafic volcanics range from 45 to 80 . Using the value for $K_{d}[(\mathrm{Fe} / \mathrm{Mg}) /(\mathrm{ol} / \mathrm{liq})]=0.30$ at $1 \mathrm{~atm}$ (see Takahashi \& Kushiro, 1983), only the most mafic Pliocene-Quaternary nephelinites and basanites and the most mafic Miocene picrites have compositions in equilibrium with mantle olivine of $\mathrm{Fo}_{88-92}$. If the 3.0-GPa value for $K_{\mathrm{d}}[(\mathrm{Fe} / \mathrm{Mg}) /(\mathrm{ol} / \mathrm{liq})]=$ 0.36 is used, then only the two most mafic Miocene picrites and one melilite nephelinite (excluding sample 1185 which contains abundant nodule debris) have compositions in equilibrium with olivine of $\mathrm{Fo}_{88-90}$.

Both the Miocene and Pliocene-Quaternary mafic volcanics exhibit systematic variations in compatible elements as a function of $\mathrm{SiO}_{2}$ content (Fig. 4). For a given $\mathrm{MgO}$ content, the picrites-tholeiites have the highest abundances of $\mathrm{Ni}, \mathrm{Cr}$, and $\mathrm{Co}$; the alkali basalts and basanites have intermediate abundances; and the nephelinites have the lowest abundances. $\mathrm{Ni}, \mathrm{Cr}$, and $\mathrm{Co}$ decrease for a given rock type with decreasing $\mathrm{MgO}$, except for the Pliocene tholeiites. Although the $\mathrm{MgO}$ content of the Pliocene tholeiites varies from 10.2 to $6.3 \mathrm{wt} . \%$, all compatible trace elements remain nearly constant with decreasing $\mathrm{MgO}$. Of the elements analyzed, only $\mathrm{Al}_{2} \mathrm{O}_{3}$ and $\mathrm{FeO}^{t}$ exhibit good correlations with $\mathrm{MgO}$ in the Pliocene tholeiites. Both oxides vary by $<10 \%$ and correlate negatively with $\mathrm{MgO}$.

With decreasing $\mathrm{MgO}$, the $\mathrm{CaO}$ content of the Miocene picrites-tholeiites and of the Pliocene-Quaternary alkali basalts and basanites initially increases gradually (Fig. 4). At $\mathrm{MgO}=7 \mathrm{wt} . \%$, the $\mathrm{CaO}$ content decreases sharply. For a given $\mathrm{MgO}$ concentration, the $\mathrm{CaO}$ concentration for both age groups increases as a function of rock type, i.e. in the sequence tholeiite-alkali basalt-basanite-nephelinite. $\mathrm{Al}_{2} \mathrm{O}_{3}$ content shows an exceptional negative correlation with $\mathrm{MgO}$ (Fig. 4). In contrast to $\mathrm{CaO}$, there are no significant differences in $\mathrm{Al}_{2} \mathrm{O}_{3}$ content as a function of rock type. With decreasing $\mathrm{MgO}$, the $\mathrm{CaO} / \mathrm{Al}_{2} \mathrm{O}_{3}$ ratio of the Miocene picrites-tholeiites decreases gradually until $\mathrm{MgO}=$ 7 wt.\%, after which it decreases sharply. The Pliocene-Quaternary tholeiites, alkali basalts, and basanites have roughly constant $\mathrm{CaO} / \mathrm{Al}_{2} \mathrm{O}_{3}$ ratios for $\mathrm{MgO}>7$ wt.\%, but below $\mathrm{MgO}=7 \mathrm{wt} . \%$, the ratio decreases with decreasing $\mathrm{MgO}$. As observed for the $\mathrm{CaO}$ content, the $\mathrm{CaO} / \mathrm{Al}_{2} \mathrm{O}_{3}$ ratio for both the Miocene and Pliocene-Quaternary volcanics increases systematically with decreasing $\mathrm{SiO}_{2}$ content of the rock group. For the Pliocene-Quaternary basalts, the ratio decreases more than a factor of two from 1.6 in the melilite nephelinites to $\sim 0-75$ in the tholeiites, which is slightly lower than the chondritic ratio of $\sim 0.9$.

For all rock types, the content of incompatible elements increases roughly with decreasing $\mathrm{MgO}$ contents (Fig. 4). At a given $\mathrm{MgO}$ content, the undersaturated mafic volcanics generally have higher concentrations of the incompatible elements.

\section{DISCUSSION}

\section{Crystal fractionation and accumulation}

Before discussing the petrogenesis of the Gran Canaria basalts, it is important to determine the effects of crystal fractionation and accumulation on the basalt chemistry. High $m g$-number and high compatible trace element abundance (see Figs. 3 and 4 and Table 1) are often used as indicators of the primary (non-differentiated) nature of basalts (e.g., Frey et al., 1978; Clague \& Frey, 1982; Clague \& Dalrymple, 1988). Although some of the Miocene tholeiites have high $m g$-number (up to 72 ), they are only in equilibrium with Fo $\leqslant 86$ (using a $K_{\mathrm{d}}$ of 0.3 for $\mathrm{Fe} / \mathrm{Mg}$ in olivine), lower than found in most mantle olivines, and thus probably none are primary compositions. Based on mass-balance calculations of the major elements, 

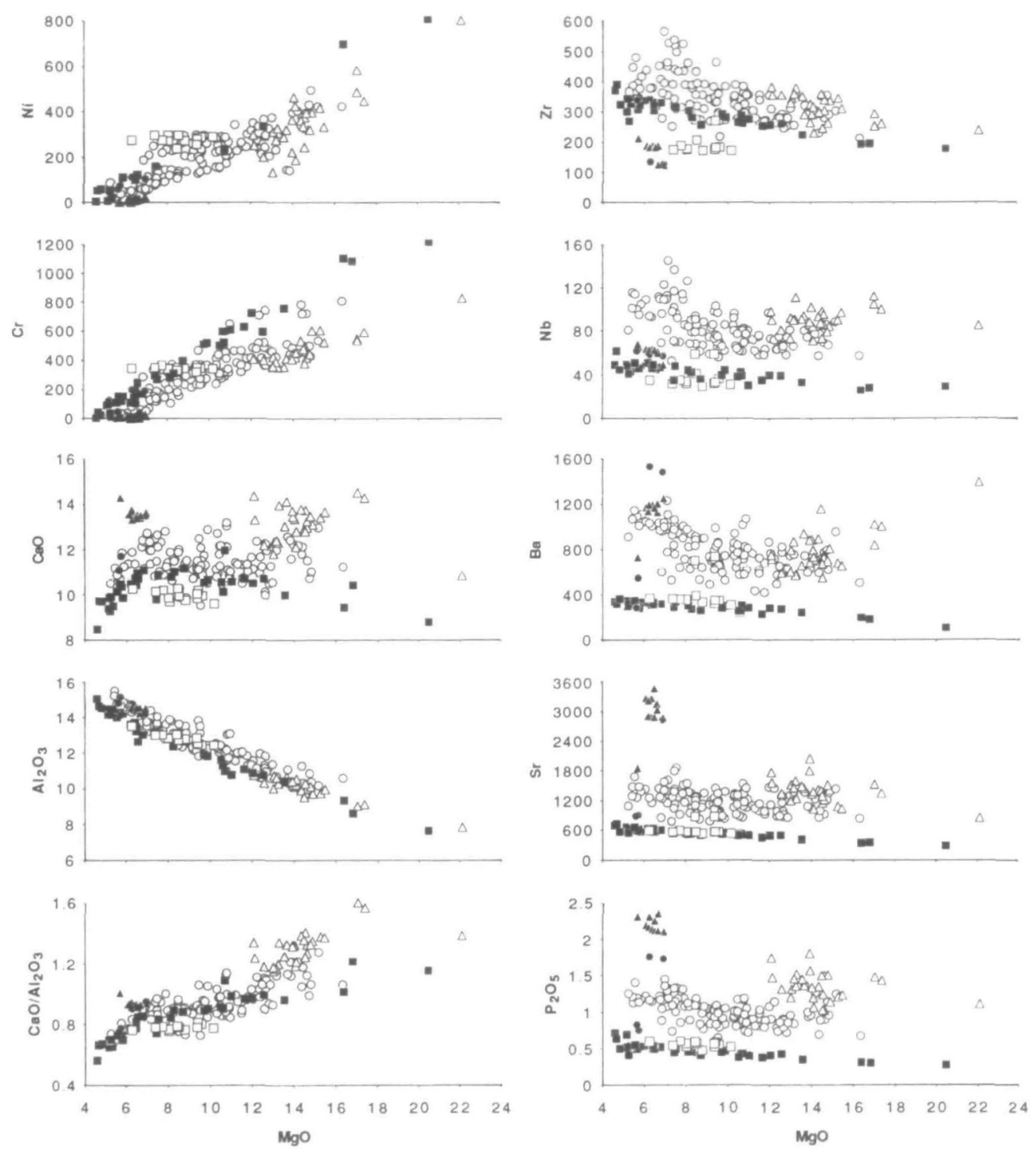

FIG. 4. Plots of $\mathrm{MgO}$ vs. compatible major and minor oxides and trace elements for the Gran Canaria mafic volcanics. (See Fig. 3 for symbols.)

the Miocene tholeiites with $\mathrm{MgO}>6 \mathrm{wt} . \%$ and the most mafic Miocene picrite (1262) can be related by removal or addition of olivine (up to $34 \%$ ) \pm clinopyroxene (up to $13 \%$ ) (Table 2 ). The elements (and oxides) incompatible in olivine, for example, $\mathrm{Al}_{2} \mathrm{O}_{3}, \mathrm{P}_{2} \mathrm{O}_{5}, \mathrm{Sr}$, and $\mathrm{TiO}_{2}$, yield excellent negative correlations with $\mathrm{MgO}$ (Fig. 4). The most incompatible elements increase roughly two-fold from the most mafic picrite to the tholeiites with $\mathrm{MgO}=6 \mathrm{wt} . \%$, consistent with removal or addition of $\sim 50 \mathrm{wt} \%$ phenocrysts. Clinopyroxene fractionation becomes important in tholeiites with $\mathrm{MgO}<7 \mathrm{wt} . \%$, as is evident by the sharp decrease in $\mathrm{CaO}, \mathrm{CaO} / \mathrm{Al}_{2} \mathrm{O}_{3}, \mathrm{CaO} / \mathrm{MgO}$, and $\mathrm{Sc}$ with decreasing $\mathrm{MgO}$ for these samples (see Fig. 4 and Table 1). The absence of either a positive or negative Eu anomaly in the rare earth element (REE) patterns (Fig. 5) and the almost two-fold variation in $\mathrm{Al}_{2} \mathrm{O}_{3}$ and $\mathrm{Sr}$ concentrations argues against substantial plagioclase fractionation or accumulation in 
TABLE 2

Summary of results from least-squares mass-balance fractional crystallization calculations

\begin{tabular}{|c|c|c|c|c|c|c|c|c|c|c|c|c|}
\hline Parent & $\begin{array}{l}\text { Rock } \\
\text { type }\end{array}$ & $\mathrm{MgO}$ & Daughter & $\begin{array}{l}\text { Rock } \\
\text { type }\end{array}$ & $\mathrm{MgO}$ & $O L$ & $C P X$ & $O X$ & $P L A G$ & $A P$ & T.S.R. & S.S.R. \\
\hline M-G-1262 & $\mathbf{P}$ & $20-5$ & M-G-1265 & $\mathrm{TH}$ & $12 \cdot 9$ & -22.9 & $-5 \cdot 9$ & & & & -28.8 & 0.15 \\
\hline M-G-1262 & $\mathbf{P}$ & $20-5$ & M-G-1528 & $\mathrm{TH}$ & $8 \cdot 1$ & $-31 \cdot 6$ & -8.6 & & & & $-40-2$ & $0-22$ \\
\hline M-G-1262 & $\mathrm{P}$ & 20.5 & $M-G-1263$ & $\mathrm{TH}$ & 6.5 & -33.9 & -12.6 & & & & -46.5 & $0-04$ \\
\hline M-G-1262 & $\mathrm{P}$ & $20-5$ & P-ET-B14 & $\mathrm{TH}$ & $10-2$ & $-29 \cdot 7$ & $-15 \cdot 6$ & & & & $-45 \cdot 3$ & 0.39 \\
\hline M-G-1262 & $\mathrm{P}$ & $20-5$ & P-ET-62.2 & $\mathrm{TH}$ & 80 & $-32 \cdot 0$ & -16.3 & & & & $-48 \cdot 3$ & $0-38$ \\
\hline M-G-1262 & $\mathrm{P}$ & $20-5$ & P-ET-63.1 & TH & $6 \cdot 3$ & $-34 \cdot 1$ & -16.7 & & & & $-50-8$ & $0-37$ \\
\hline P-ET-B14 & TH & $10-2$ & P-ET-63.1 & TH & $6 \cdot 3$ & $-10-2$ & & $1 \cdot 2$ & -1.5 & & $-10-5$ & $0-14$ \\
\hline P-RN-94 & $\mathrm{AB}$ & $12 \cdot 8$ & Q-D6 & AB & 9.6 & $-9 \cdot 7$ & -5.8 & $1 \cdot 3$ & & & -14.2 & $0-44$ \\
\hline P-RN-94 & $\mathrm{AB}$ & $12 \cdot 8$ & M-M-1721 & $\mathrm{AB}$ & $5 \cdot 6$ & -16.6 & $-8 \cdot 2$ & & & & $-24 \cdot 8$ & 0.22 \\
\hline P-RN-94 & $\mathrm{AB}$ & $12 \cdot 8$ & P-ET-83 & $\mathrm{AB}$ & $8 \cdot 8$ & $-10-6$ & $-1 \cdot 4$ & 10 & & & -11.0 & $0-36$ \\
\hline P-RN-94 & $\mathrm{AB}$ & $12 \cdot 8$ & P-RN-70 & $\mathrm{AB}$ & $5 \cdot 5$ & $-19 \cdot 1$ & $-11 \cdot 5$ & & & & $-30-6$ & $0-36$ \\
\hline P-ET-90 & B & $12 \cdot 7$ & P-ET-52 & B & $8 \cdot 5$ & $-10-8$ & & & & & $-10-8$ & 0.37 \\
\hline P-LL-1138 & B & $14 \cdot 4$ & P-RN-B129 & B & 7.9 & $-15 \cdot 6$ & -9.7 & & & & $-25 \cdot 3$ & $0-36$ \\
\hline P.RN-1130 & B & $12 \cdot 4$ & P-RN-109 & $\mathbf{T}$ & $5 \cdot 3$ & $-15-0$ & $-29 \cdot 1$ & $-3 \cdot 4$ & & -0.5 & $-48 \cdot 0$ & 0.27 \\
\hline P-LL-1115 & $\mathbf{N}$ & $13 \cdot 7$ & $M-F-683$ & $\mathbf{N}$ & $6 \cdot 7$ & $-17 \cdot 1$ & $-15 \cdot 1$ & $1 \cdot 8$ & & & $-30-4$ & $0-35$ \\
\hline
\end{tabular}

Parent and daughter compositions are in Table 1, except for tephrite sample 109 (Hoernle, 1987). Sample numbers are prefixed by abbreviations for the volcanic cycle (MMiocene, P-Pliocene, Q-Quaternary) and formation (same abbreviations as used in Table 1). Rock type abbreviations are the same as in Table 1, plus T-tephrite. The MgO values were recalculated on a volatile-free basis with $\mathrm{Fe}^{3+} /\left(\mathrm{Fe}^{3+}+\mathrm{Fe}^{2+}\right)=0-2$. Contents of minerals are in weight percent and refer to the amount of the mineral subtracted (negative sign) or added (no sign). Mineral abbreviations are as follows: OL-olivine (Fo ${ }_{79-83}$ ), CPX-clinopyroxene (titanaugite-acmitic augite), OX-(magnetite-ilmenite), PLAG-plagioclase $\left(A n_{79}\right)$, AP-apatite. Mineral compositions are from the Pliocene basalts (Hoernle, 1987), except for plagioclase whıch is from Miocene tholeiite flow T1 (Crisp \& Spera, 1987). T.S.R. refers to the total solids removed from the parent to derive the daughter. If S.S.R. (sum of the squares of the residuals) < 1, then a basalt pair may be related by fractional crystallization or accumulation. 

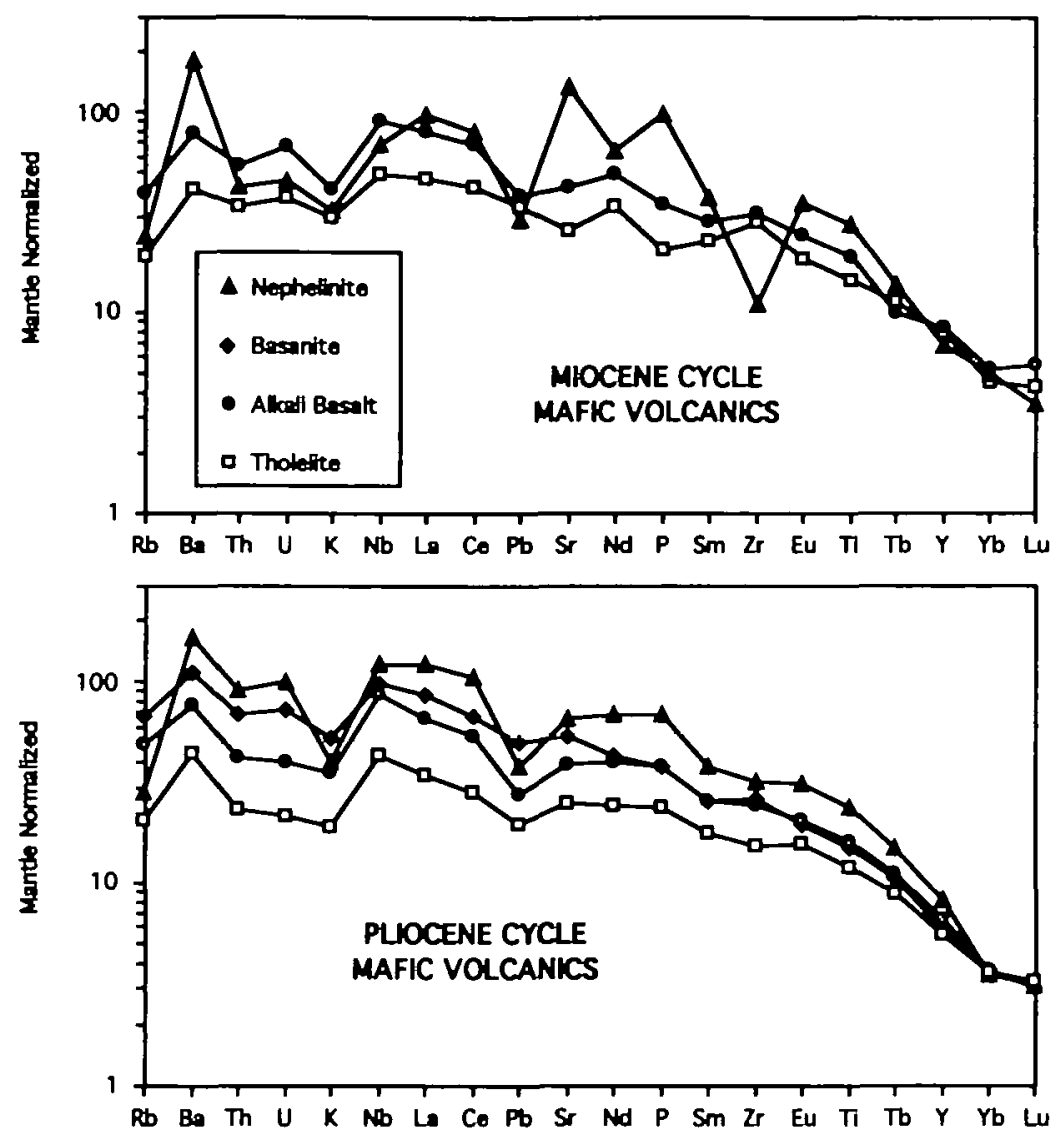

FIG. 5. Plots of incompatible trace elements normalized to primitive mantle (after Sun \& McDonough, 1989) for Miocene and Pliocene Cycle mafic volcanics. The overlapping HREE abundances are consistent with the presence of garnet in both the Miocene and Pliocene-Quaternary sources. The increase in most incompatible elements from tholeiite to alkali basalt to basanite to nephelinite is consistent with decreasing degrees of partial melting in this sequence. The low abundances of $K$ and $R b$ in the nephelinites from both cycles suggests that a phase such as phlogopite or amphibole remains in the residuum at low degrees of partial melting. (Note the anomalous pattern for the Miocene nephelinite.)

samples with $\mathrm{MgO}>6$ wt.\%. Plagioclase, $\mathrm{Fe}-\mathrm{Ti}$ oxides, and apatite are present as phenocrysts in samples with $\mathrm{MgO}<6 \mathrm{wt} . \%$. Mass-balance calculations (Cousens $e t$ al., 1990) and variations in the trace elements require removal of these phases to derive these samples from samples with $\mathrm{MgO}>6 \mathrm{wt} . \%$. The absence of plagioclase phenocrysts and of evidence requiring plagioclase fractionation or accumulation and the presence of clinopyroxene in the picrites and tholeiites with $\mathrm{MgO}>6 \mathrm{wt} . \%$ suggests that fractionation and/or accumulation in these samples occurred at depths $>15 \mathrm{~km}$ (Albarede \& Tamagnan, 1988 , and references therein) or during transport of these magmas through the lithospheric mantle, as the Moho beneath Gran Canaria is at $\sim 15 \mathrm{~km}$ (Banda et al., 1981). The presence of plagioclase on the liquidus for the Miocene tholeiites with $\mathrm{MgO}<6 \mathrm{wt} \%$ indicates that fractionation probably occurred at the base of or within the crust.

Based on the abundance and size of olivine and clinopyroxene phenocrysts in the Miocene picrites, their whole-rock compositions clearly do not reflect liquid compositions but rather a basalt-crystal mixture. The major and trace element data for the Miocene picrite-tholeiite 
suite form continuous trends (Fig. 4), consistent with a genetic relationship between these rock types. Two possible origins for the picrites are (1) accumulation of olivine \pm clinopyroxene during crystal fractionation (i.e., the crystals are genetically related to the basaltic groundmass) or (2) incorporation of olivine \pm clinopyroxene cumulates from earlier melts (i.e., crystals are xenocrystic) (Schmincke, 1982). Partial resorption of olivine and, less commonly, clinopyroxene phenocrysts and the occurrence of these phases as glomerocrysts in the Miocene picrites suggest a secondary, or possibly cumulus, origin for at least some of these phenocrysts. As the $m g$-numbers of the tholeiites are too low for them to be primary compositions, and as olivine is the major fractionating phase in the tholeiites, accumulation of fractionating olivine in or addition of xenocrystic olivine to a tholeiite will have a similar effect on the magma composition, serving to shift it back to the primary or near-primary melt composition. Therefore, although the picrites probably represent slightly fractionated compositions which have incorporated cumulus olivine \pm clinopyroxene, the whole-rock compositions of the picrites are probably similar to the parental compositions of the tholeiites. A more detailed study of the picrites is under way.

The Pliocene tholeiites, with $m g$-numbers ranging from 52 to 65 , are more restricted in composition than the Miocene tholeiites. The nearly identical isotopic composition of the five Pliocene tholeiites analyzed for isotopes (Hoernle et al., 1991) suggests that they were derived from a single parent or source. Although the most evolved Pliocene tholeiite (sample 63.1) can be derived from the most mafic Pliocene tholeiite (sample B14) by removal of $10 \%$ olivine and $1.5 \%$ plagioclase, and addition of $1.2 \%$ titanomagnetite (Table 2), olivine fractionation is inconsistent with the nearly constant $\mathrm{Cr}, \mathrm{Co}$, and $\mathrm{Ni}$ concentrations in the Pliocene tholeiites. For example, B14 has $340 \mathrm{ppm} \mathrm{Cr}, 51 \mathrm{ppm} \mathrm{Co}$, and $290 \mathrm{ppm} \mathrm{Ni}$, compared with $345 \mathrm{ppm} \mathrm{Cr}, 57 \mathrm{ppm} \mathrm{Co}$, and $276 \mathrm{ppm} \mathrm{Ni}$ in sample 63.1 (also see Fig. 4 and Table 1). Therefore fractional crystallization cannot alone relate the different Pliocene tholeiites.

Using a $K_{\mathrm{d}}$ of 0.3 for $\mathrm{Fe} / \mathrm{Mg}$ in olivine, the Pliocene tholeiites are only in equilibrium with olivines of $\mathrm{Fo}_{74-83}$, too low for most mantle compositions, and therefore probably do not reflect primary melts. Subtraction of $30-34 \%$ olivine and $15-17 \%$ clinopyroxene from the most mafic Miocene picrite (sample 1262, which has a composition that could have been in equilibrium with a mantle assemblage) can yield the range in major elements observed for the Pliocene tholeiites (Table 2). Nonetheless, the roughly constant (1) compatible element $(\mathrm{Cr}$, $\mathrm{Co}, \mathrm{Ni}, \mathrm{V}$, and $\mathrm{Sc}$ ) concentrations and (2) $\mathrm{CaO} / \mathrm{Al}_{2} \mathrm{O}_{3}, \mathrm{CaO} / \mathrm{Na}_{2} \mathrm{O}$, and $\mathrm{Sc} / \mathrm{Yb}$ ratios (see Fig. 4 and Table 1) in the Pliocene tholeiites are not consistent with derivation from a single parent composition. Therefore the similarity in isotopic composition of the Pliocene tholeiites suggests derivation from similar source material but under different conditions of melting, i.e. temperature, pressure, volatile content, etc. These parental melts then underwent fractional crystallization to produce the Pliocene tholeiites.

The Miocene alkali basalts and basanites and the Pliocene-Quaternary alkali basalts also represent evolved compositions (Fig. 3). The Pliocene-Quaternary basanites, which are in equilibrium with $\mathrm{Fo}_{75-89}$ using a 1-bar $K_{\mathrm{d}}$ or with $\mathrm{Fo}_{71-87}$ using a 3.0-GPa $K_{\mathrm{d}}$ (Takahashi \& Kushiro, 1983), range from near-primary compositions to somewhat evolved compositions. The roughly constant $\mathrm{CaO} / \mathrm{Al}_{2} \mathrm{O}_{3}$ and $\mathrm{CaO} / \mathrm{Na}_{2} \mathrm{O}$ ratios for the Pliocene-Quaternary alkali basalts and basanites argue against significant clinopyroxene fractionation for samples with $\mathrm{MgO}>8$. The sharp decrease in $\mathrm{CaO}$ and $\mathrm{Sc}$ concentrations and of the $\mathrm{CaO} / \mathrm{Al}_{2} \mathrm{O}_{3}$ and $\mathrm{CaO} / \mathrm{Na}_{2} \mathrm{O}$ ratios in samples with $\mathrm{MgO}<8$ (Table 1, Fig. 4) indicate that clinopyroxene fractionation begins to play an important role in these rocks, as is evident from the mass balance calculations (Table 2). The increase in the incompatible elements with decreasing $\mathrm{MgO}$ (Fig. 4) and the mass-balance solutions (Table 2) are consistent with removal of 
30-50 wt.\% of mafic phenocrysts from the basalts with the highest $\mathrm{MgO}$ to derive those with the lowest $\mathrm{MgO}$. Miocene alkali basalt can be derived from near-primary Pliocene alkali basalt through removal of olivine, clinopyroxene $\pm \mathrm{Fe}-\mathrm{Ti}$ oxides, and apatite (Table 2 ). Plagioclase becomes an important fractionating phase only in alkali basalts and basanites with $\mathrm{MgO}<5-6$ (Hoernle, 1987). Experimental data on melts with similar compositions to the Gran Canaria alkali basalts indicate that olivine is followed by clinopyroxene, instead of plagioclase, at pressure of $\sim 0.8 \mathrm{GPa}$ (Mahood \& Baker, 1986; Fisk et al., 1988). These data suggest that fractionation in the alkalic basalts and possibly basanites occurred at depths of $>25 \mathrm{~km}$ or within the mantle.

The Miocene nephelinites have $m g$-numbers of 48 and 53, and thus are moderately evolved. To explain the 'extraordinarily' high contents of $\mathrm{TiO}_{2}, \mathrm{P}_{2} \mathrm{O}_{5}$, and $\mathrm{Sr}$, Cousens et al. (1990) suggested that sample 683 has accumulated apatite and titanomagnetite, which are present only as groundmass phases. After removing apatite and titanomagnetite until the $\mathrm{P}_{2} \mathrm{O}_{5}$ and $\mathrm{TiO}_{2}$ contents are 'typical of Gran Canaria basalts', they proposed a parental composition for 683 which contains $43 \% \mathrm{SiO}_{2}, 15 \% \mathrm{Al}_{2} \mathrm{O}_{3}, 15 \% \mathrm{Fe}_{2} \mathrm{O}_{3}, 7 \% \mathrm{MgO}$, etc., and has an $m g$-number of 54 . The $m g$-number of the proposed parental composition is too low for it to be a primary or near-primary composition. Furthermore, the small positive Eu anomaly of this nephelinite (Table 1), if not a source characteristic, suggests apatite fractionation rather than accumulation (Hanson, 1980; Peterson, 1989). Alternatively, the major element compositions of the Miocene nephelinites could be derived from the major element compositions of Pliocene nephelinites through removal of olivine and clinopyroxene, which are phenocryst phases, and the removal or addition of $\mathrm{Fe}-\mathrm{Ti}$ oxides (see example in Table 2). Removal of 30-35 wt.\% of olivine and clinopyroxene, as is indicated by the mass-balance calculations, can help explain the high contents of some of the oxides and elements incompatible in these phases, such as, $\mathrm{P}_{2} \mathrm{O}_{5}, \mathrm{Sr}$, and $\mathrm{TiO}_{2}$.

Fractionation of these phases, however, cannot explain why the Miocene nephelinites and basanites have much lower contents of $\mathrm{K}_{2} \mathrm{O}$, light REE (LREE), Rb, $\mathrm{Zr}, \mathrm{Hf}, \mathrm{Nb}, \mathrm{Ta}, \mathrm{Th}, \mathrm{U}$, and $\mathrm{Pb}$ than the Pliocene basanites and nephelinites (see Fig. 5 and Table 1). One possible way of decreasing the concentrations of these normally incompatible elements without significantly affecting the concentrations of the major elements is through fractionation of minor phases, such as phlogopite and zircon. Fractionation of these phases, however, seems unlikely, as neither phase has ever been observed in any of the Gran Canaria mafic volcanics. Furthermore, experimental studies indicate that zircon is saturated in basaltic melts only when $\mathrm{Zr}$ contents are 2-3 wt.\% (Watson, 1979; Watson \& Harrison, 1983; Irving \& Frey, 1984).

All of the Pliocene-Quaternary nephelinites have $m g$-number $>66$ and $\mathrm{Ni}>130$, with all but four having $\mathrm{Ni}>245$. The nephelinites are in equilibrium with $\mathrm{Fo}_{84-92}$ at 1 bar and with $\mathrm{Fo}_{82-90}$ at $3.0 \mathrm{GPa}$ (Takahashi \& Kushiro, 1983), and range from primary or near-primary to slightly evolved compositions, having lost only minor amounts of olivine $(<15 \%)$. Some of the nephelinites contain spinel lherzolite xenoliths, further indicating that they are primary or near-primary. The anomalously high $m g$-number $(81)$ and Ni concentration $(805 \mathrm{ppm})$ of melilite-bearing nephelinite 1185 reflects the abundance of nodule debris in this sample.

\section{Depth of melting}

Estimates of the depths at which basaltic melts are generated provide important constraints on the petrogenesis of basalts and ultimately on the origin of ocean island volcanoes. We have used two geochemical methods to estimate the depths of melting. The 
first method is based on the comparison of the inferred source mineralogy with the experimentally determined stability range of this mineral assemblage. The second method involves comparing the major-element chemistry of primitive basalts with the results of experimental melting studies. The behavior of major and trace elements which are compatible during at least part of the melting interval can yield valuable information on the source mineralogy. Both the Miocene and Pliocene-Quaternary mafic volcanics have nearly constant $\mathrm{Al}_{2} \mathrm{O}_{3}, \mathrm{Sc}$, and $\mathrm{Y}$ abundances with respect to $\mathrm{MgO}$, and overlapping heavy REE (HREE) abundances ranging from 5 to 10 times chondrite in the most mafic volcanics $(\mathrm{mg}$ number $\geqslant 65$ ) and in the estimated parental compositions for the more evolved volcanics ( $m g$-number $<65$ ) (see Table 1 and Figs. 4 and 5). These variations are consistent with residual garnet in both the Miocene and Pliocene-Quaternary sources (Schmincke, 1982). Garnet-bearing sources probably require depths in excess of $\sim 80 \mathrm{~km}$ (e.g., McKenzie \& O'Nions, 1991). Low $\mathrm{K}_{2} \mathrm{O}, \mathrm{Rb}$, and $\mathrm{Ba}$ contents in the Miocene and Pliocene-Quaternary basanites and nephelinites, and the absence of a positive correlation between these elements and other highly incompatible elements, such as $\mathrm{P}_{2} \mathrm{O}_{5}$ and $\mathrm{Sr}$, suggest the presence of a residual $\mathrm{K}$-phase in the sources of these undersaturated mafic volcanics (see Schmincke \& Flower, 1974; Hoernle \& Schmincke, this volume). Lower $\mathrm{Ba} / \mathrm{Sr}$ and $\mathrm{K} / \mathrm{Nb}$ ratios in these mafic volcanics, as compared with the alkali basalts and tholeiites, argue for generation of these magmas under $\mathrm{CO}_{2}$-rich conditions in the presence of residual phlogopite instead of amphibole (Clague \& Frey, 1982; Hawkesworth et al., 1990). A lherzolitic mantle assemblage containing garnet, phlogopite, and a carbonate phase is stable only at pressures in excess of $2.9 \mathrm{GPa}$ or depths of $\sim 100 \mathrm{~km}$ (Brey et al., 1983).

Experimental studies provide a database from which the depth of melting of primary or near-primary compositions can be estimated (Takahashi \& Kushiro, 1983; Takahashi, 1986; Falloon \& Green, 1987, 1988; Falloon et al., 1988). The cation mol \% MgO vs. FeO' diagram (Fig. 6) is useful in estimating depths of melting, especially as olivine fractional crystallization or accumulation and extent of melting will not significantly modify the $\mathrm{FeO}^{\prime}$ mol\% (Langmuir \& Hanson, 1980). The $\mathrm{FeO} / \mathrm{MgO}$ ratio (or fertility) of the source, however, can affect the composition of the melts and must be taken into consideration. Figure 6 contains data for the Gran Canaria basalts and experimental data from high-pressure melting studies (2.0-3.5 GPa) of dry and fertile initial bulk compositions, which have been proposed as source compositions for ocean island magmas [starting compositions are Hawaiian Pyrolite, i.e., Pyrolite III $-40 \%$ olivine (Falloon et al., 1988), MPY-87, i.e., MORB Pyrolite recalculated to $m g$-number 87 from 90 (Falloon \& Green, 1988), and spinel Iherzolite HK66 from Salt Lake Crater, Hawaii (Takahashi \& Kushiro, 1983)]. To avoid the problems of quench modification of primary liquids and of reactions between the capsules and the melt, which can affect the oxygen fugacity and the Fe content of the melt (Takahashi \& Kushiro, 1983; Falloon \& Green, 1987, 1988; Falloon et al., 1988), we have included in Fig. 6 only data from runs made in graphite or graphite-lined platinum capsules and for which the basalt-peridotite 'sandwich' technique was used. As was demonstrated at lower pressures (0.5-1.5 GPa) by Klein \& Langmuir (1987), the high-pressure experimental data (2.0-3.5 GPa) also show that both $\mathrm{FeO}^{\mathrm{t}}$ and $\mathrm{MgO}$ increase with increasing pressure of melting. The magnitude of the pressure dependence agrees well with that calculated by Langmuir \& Hanson (1980).

The Canary magmas have high $\mathrm{FeO}^{\mathrm{t}}$ contents (Schmincke, 1982), which are similar to or up to $3.5 \mathrm{~mol} \%$ higher than the melts equilibrated with fertile lherzolite compositions at $3.0 \mathrm{GPa}$. The higher $\mathrm{FeO}^{\mathrm{t}}$ could reflect either $\mathrm{FeO} / \mathrm{MgO}$ ratios of up to $60 \%$ greater in the Gran Canaria source than in the compositions used for the experiments, or depths of melting in excess of 3.0 GPa for the Gran Canaria basalts (Langmuir \& Hanson, 1980). As the most 


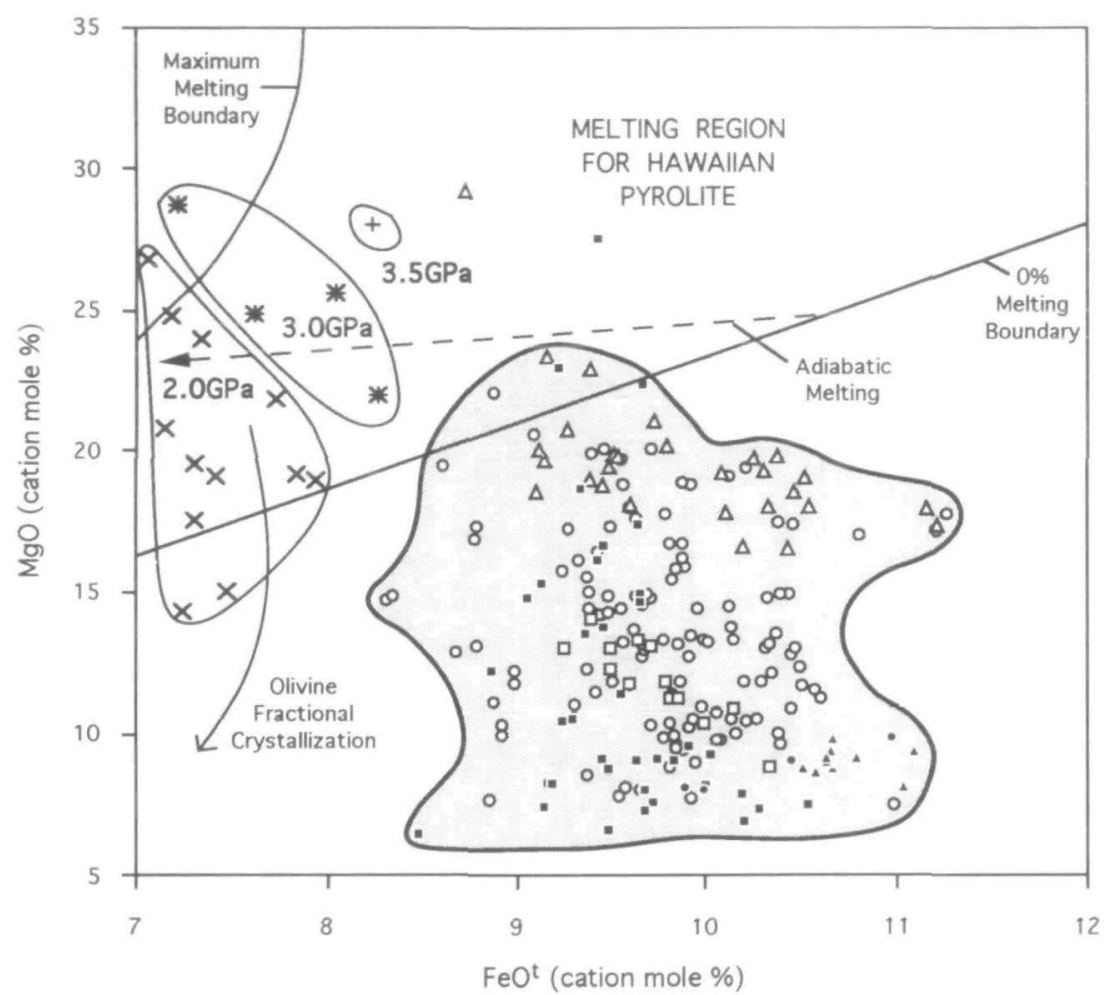

FIG. 6. Cation mole percentage of FeO vs. MgO (modified from Langmuir \& Hanson, 1980). The high total FeO $\mathrm{mol} \%$ for the Gran Canaria mafic volcanics indicates that they were formed at pressures $>30 \mathrm{GPa}$. The results from melting experiments on fertile mantle compositions at pressures of $2-0,3 \cdot 0$, and $3.5 \mathrm{GPa}$ are from Takahashi \& Kushiro (1983), Fallon \& Green (1988), and Falloon et al. (1988). The melting region for Hawaiian pyrolite (between the $0 \%$ and maximum melting boundaries), a liquid line of descent for fractional crystallization of olivine, and a possible adiabatic melting path are modified from Klein \& Langmuir (1987). Symbols for Gran Canaria mafic volcanics are the same as in Fig. 3.

mafic Gran Canaria basalts are in equilibrium with olivine having similar or slightly higher $m g$-number than the initial bulk compositions used in the melting experiments, $\mathrm{FeO} / \mathrm{MgO}$ ratios of up to $60 \%$ higher are unreasonable if the most mafic Gran Canaria basalt compositions reflect primary or near-primary melts. Both the Miocene and Pliocene cycles of volcanism nearly cover the whole range in $\mathrm{FeO}^{\prime}$ wt.\% observed for Gran Canaria, indicating that the different cycles of volcanism originated from similar depths. Although there is much overlap, the most mafic, $\mathrm{SiO}_{2}$-undersaturated basalts extend to higher $\mathrm{FeO}^{\mathfrak{l}}$ contents than the most mafic, $\mathrm{SiO}_{2}$-saturated basalts, which could reflect derivation of the undersaturated basalts from generally greater depths or from more fertile source material. The negative slope of the Pliocene tholeiites, which are isotopically nearly identical but cannot be related through crystal fractionation processes alone, may reflect melting at different depths and to different degrees.

The projection from diopside onto the base of the CIPW normative basalt tetrahedron (Fig. 7) is also useful for evaluating the effects of pressure on basalt composition (Jacques \& Green, 1980; Takahashi \& Kushiro, 1983; Falloon \& Green, 1988; Falloon et al., 1988). With decreasing pressure, the dry olivine + orthopyroxene + clinopyroxene cotectics (from 0 to $8.0 \mathrm{GPa}$ ) for a wide variety of starting compositions move systematically away from the olivine apex. The 5.0- and 8-0-GPa cotectics shown in Fig. 7 are based on one melt 
composition each (Takahashi, 1986). As hypersthene was not detected in the residue for either sample, the melt compositions may have started moving towards olivine and thus are approximate at best. Saturation of the melts with either $\mathrm{H}_{2} \mathrm{O}$ or $\mathrm{CH}_{4}$ will also shift the cotectics away from the olivine apex (Green et al., 1987). Increasing pressure, $\mathrm{C}, \mathrm{CO}_{2}$ and/or $\mathrm{CO}_{3}$ saturation, and olivine accumulation will shift the cotectics towards the olivine apex. Olivine fractionation will move the melt composition directly away from the olivine apex.

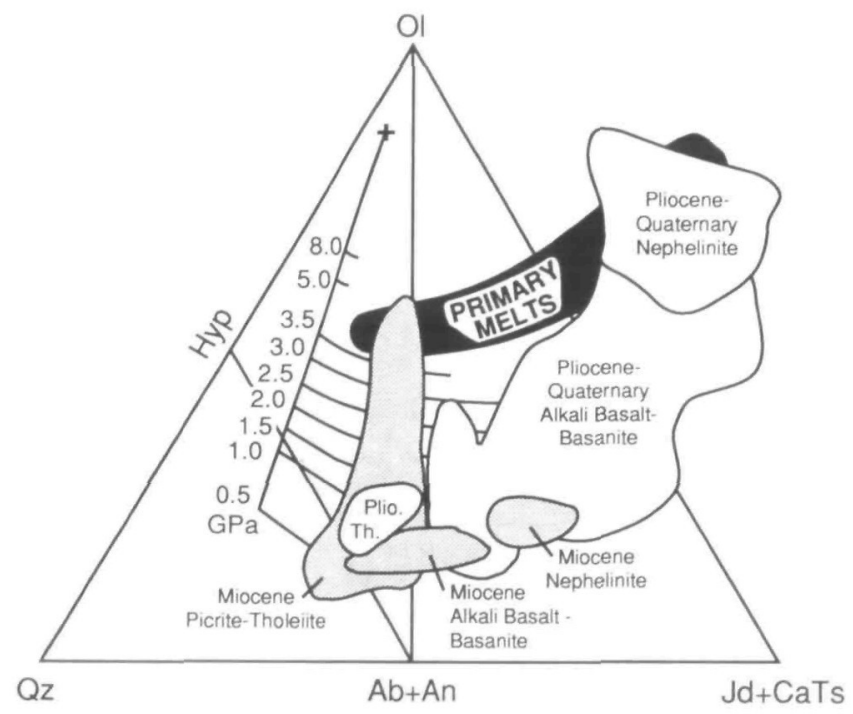

FIG. 7. Projection from diopside (Di) onto the base of the CIPW molecular normative basalt tetrahedron: olivine (Ol)-quartz (Qz)-jadeite plus calcium Tschermak's molecule (Jd + CaTs) (modified from Falloon \& Green, 1988; Falloon et al., 1988). Hyp-hypersthene, Ab-albite, An-anorthite. The lines which are roughly parallel to the $\mathrm{Qz}-(\mathrm{Jd}+\mathrm{CaTs})$ join are $\mathrm{Ol}+\mathrm{Hyp} \pm \mathrm{Cpx} \pm \mathrm{Gt}$ cotectics defined by partial melt compositions at 0-5-8-0 GPa under anhydrous conditions (Takahashi \& Kushiro, 1983; Takahashi, 1986; Falloon \& Green, 1987, 1988; Falloon et al., 1988). The line roughly parallel to the $\mathrm{Ol}+\mathrm{Qz}$ join represents an Ol-only cotectic. The 'primary melt' field was derived by adding olivine of appropriate composition to all basalt compositions with $\mathrm{MgO}>8$ until they were in equilibrium with mantle olivine $\left\{\mathrm{Fo}_{88-91}\right.$; using a 3-0-GPa $K_{\mathrm{d}}[(\mathrm{Fe} / \mathrm{Mg}) /(\mathrm{ol} / \mathrm{liq})]=0-36$ from Takahashi \& $\mathrm{Kushiro}$ (1983)\}. CIPW norm was calculated using $\mathrm{Fe}^{2+} /\left(\mathrm{Fe}^{2+}+\mathrm{Fe}^{3+}\right)=09$, in keeping with Falloon \& Green (1988).

When significant amounts of clinopyroxene join olivine as an important fractionating phase, the melt composition will move towards the $\mathrm{Qz}$ apex. For isobaric melting, the composition of the melts will move along a given cotectic towards its source composition (the ' + ' in Fig. 7 represents one possible source composition), as the extent of melting increases. As the fertility (i.e., Jd $+\mathrm{CaTs}$ or Nepheline $+\mathrm{CaAl}_{2} \mathrm{O}_{4}$ component) in the source increases, the composition of the initial melts will move increasingly farther from the $\mathrm{Ol}-\mathrm{Qz}$ join along one of the cotectics for isobaric melting.

The Miocene picrites-tholeiites form an elongate field parallel to olivine control lines (Fig. 7). The most primitive picrites plot between the 3.5- and 5.0-GPa cotectics, whereas the most evolved tholeiites fall below the 0-5-GPa cotectic. The Miocene picrite-tholeiite trend illustrates how olivine \pm clinopyroxene crystal fractionation can drive the composition of a picrite away from the olivine apex and into the quartz normative region of the triangle. Conversely, olivine accumulation can shift the bulk composition of a tholeiitic melt, formed at $<2.0-2.5 \mathrm{GPa}$, towards the olivine apex. The Pliocene tholeiites overlap the Miocene tholeiite field and fall between the 0.5- and 1.5-GPa dry cotectics, consistent with their 
derivation through crystal fractionation of a more mafic parent formed at high pressure or as primary melts formed at $0.5-1.5 \mathrm{GPa}$. The Hawaiian picrites-tholeiites (not shown) form a field almost identical to that of the Gran Canaria picrites-tholeiites, except that the Hawaiian field extends to only the 3.0 GPa cotectic (see Falloon et al., 1988, fig. 8). Recently, glasses containing up to $15 \mathrm{wt} . \% \mathrm{MgO}$ have been discovered in sands offshore of Kilauea, demonstrating that primary Hawaiian tholeiites must contain $>15 \mathrm{wt} . \% \mathrm{MgO}$ (Clague \& Weber, 1991). The highest estimates of the primary composition of Hawaiian tholeiites range from 17 wt.\% MgO (Clague \& Weber, 1991) to 20-25 wt.\% MgO (Wright, 1984). If the olivine phenocrysts and tholeiitic groundmass are genetically related, then the most mafic Miocene picrites may represent primary to near-primary compositions. Under dry conditions, these compositions would have formed by melting at depths $>3.5 \mathrm{GPa}$, in excellent agreement with depth estimates based on their FeO' contents (Fig. 6). Nonetheless, even if the picrites were formed by incorporation of xenocrystic (cumulus) olivine into a more evolved, tholeiitic melt, their (as well as the tholeiites') parental magmas still must have formed at depths $>3.0 \mathrm{GPa}$ if they originated from a garnet-bearing source as discussed above, as 'a garnet-bearing cotectic at pressures $\leqslant 3.0 \mathrm{GPa}$ would lie to the (right) of the Ab-An-Ol join' in Fig. 7 (Falloon et al., 1988).

Almost all of the Miocene and Pliocene-Quaternary basalts have undergone some olivine \pm clinopyroxene crystallization. Based on the mass-balance calculations and trace element variations, samples with $\mathrm{MgO}>8$, however, probably have lost $<5-10 \%$ clinopyroxene phenocrysts. Therefore we can estimate the parental compositions of the evolved basalts by adding olivine of appropriate composition until these samples are in equilibrium with mantle olivines of $\mathrm{Fo}_{88-90}$. When this is done, the samples with $\mathrm{MgO}>8$ plot above the 3.5-GPa cotectic in the field labeled 'primary melt' in Fig. 7, further supporting pressures of melting $>3.0-3.5 \mathrm{GPa}$.

The three methods outlined above each indicate pressures of magma generation beneath Gran Canaria in excess of 3.0-3.5 GPa. For oceanic lithosphere formed $>150 \mathrm{Ma}$, such as that beneath Gran Canaria, the mechanical boundary layer thickness is $\sim 100 \mathrm{~km}$ (equivalent to pressures of $\sim 3.0 \mathrm{GPa}$ ) (Zhang \& Tanimoto, 1991). Therefore the depths estimated from the major and trace element data are consistent with melting occurring by decompression within an upwelling column of asthenospheric (plume) material which encounters a mechanical boundary layer at $\sim 100 \mathrm{~km}$ beneath the Canary Islands.

As has been shown for mid-ocean ridge basalts (MORB) (Klein \& Langmuir, 1987; McKenzie \& Bickle, 1988), much of the major and minor element variations in ocean island basalts (OIB), as well as the melt production rate, may also reflect the length of the melting interval. The temperature (possibly related to the buoyancy flux) and composition of the upwelling plume material will determine the depth at which melting begins, whereas the thickness of the mechanical boundary layer will define the upper limit of the melting interval.

Watson \& McKenzie (1991) showed that melt production is strongly dependent on the upper boundary of the melting column, and proposed that the thickness of the mechanical boundary layer is the primary control on the melt production rate. Recent seismic studies indicate that the mechanical boundary layer (lithosphere) thickens continuously with age to $\sim 150 \mathrm{Ma}$, instead of 60-80 Ma as previously believed (Zhang \& Tanimoto, 1991). Therefore we would expect a difference in the melt production rates between Hawaii, situated on crust formed at $\sim 90 \mathrm{Ma}$, and the Canaries, on crust formed at $>160 \mathrm{Ma}$. Using a mechanical boundary layer thickness of $72 \mathrm{~km}$ for Hawaii (in good agreement with the $75-\mathrm{km}$ thickness estimated by the method of Zhang \& Tanimoto) and a melting interval that extends from this depth to $136 \mathrm{~km}$, Watson \& McKenzie were able to derive the observed melt production rate for the Hawaiian shield stage of $0.16 \mathrm{~km}^{3} / \mathrm{yr}$. For a mechanical boundary layer thickness of 
$\sim 110 \mathrm{~km}$, probably slightly deeper than that beneath the Canary Islands, they estimated a production rate of $0.015 \mathrm{~km}^{3} / \mathrm{yr}$. Although the duration of the shield-building volcanism on Gran Canaria is at present poorly constrained, we can estimate an average melt production rate for the entire Canary archipelago. The average melt production rate ranges from 0.0017 to $0.0068 \mathrm{~km}^{3} / \mathrm{yr}$ depending on whether $80 \mathrm{Ma}$ (Le Bas et al., 1986b) or $20 \mathrm{Ma}$ (McDougall \& Schmincke, 1976), respectively, is used for the age of the archipelago. These rates are considerably lower than the estimate of Watson \& McKenzie. If we assume, however, that the volcanic edifices represent only $20 \%$ of the melt generated in the plume (and intrusives the other $80 \%$ ), as they did for Hawaii, the estimated melt production rates $\left(0.0084-0.034 \mathrm{~km}^{3} / \mathrm{yr}\right)$ agree well with the value they calculated for a $110 \mathrm{~km}$ thick mechanical boundary layer.

As a result of the large uncertainties in the age of the Canary Islands and in the volume of intrusives, it is, however, difficult to assess if the lithospheric thickness is the primary factor controlling the low melt production rates for the Canaries. The low buoyancy flux $(1 \mathrm{Mg} / \mathrm{s}$, estimated for the Canary and Madeira Hotspots combined; Sleep, 1990) no doubt also contributes to the low melt production rate. The melting interval for the Canary Plume is probably one of the shortest for plumes beneath oceanic lithosphere, as a result of the thick lithosphere beneath the Canaries and the low buoyancy flux of the Canary Plume.

\section{CONCLUSIONS}

Miocene to Recent basalts on Gran Canaria range from near-primary or primary compositions to evolved compositions with possibly up to $50 \%$ mafic phases removed. The major and trace element data for the Miocene and Pliocene tholeiites are consistent with their derivation from picritic compositions $(\mathrm{MgO}=20-22 \mathrm{wt} . \%)$ through olivine \pm minor clinopyroxene fractionation. The Miocene tholeiites have compositions consistent with their derivation from magmas similar to the Miocene picrites. The compositions of the Pliocene tholeiites preclude their origin from a single parent composition and probably reflect different degrees of melting at different depths. The most evolved Gran Canaria alkali basalts, basanites, and nephelinites can be derived from primitive Pliocene-Quaternary alkali basalts, basanites, and nephelinites through subtraction of olivine \pm clinopyroxene, $\mathrm{Fe}-\mathrm{Ti}$ oxides, plagioclase, and apatite. The low concentrations of $\mathrm{K}_{2} \mathrm{O}, \mathrm{LREE}, \mathrm{Rb}, \mathrm{Zr}, \mathrm{Hf}$, $\mathrm{Nb}, \mathrm{Ta}, \mathrm{Th}, \mathrm{U}$, and $\mathrm{Pb}$ in the Miocene basanites and nephelinites are believed to reflect lower concentrations of these elements in their parental magmas, as compared with Pliocene-Quaternary basanites and nephelinites. The presence of clinopyroxene and absence of plagioclase on the liquidus in the Miocene tholeiites and in the Pliocene-Quaternary alkali basalts and basanites with $\mathrm{MgO}>6$ suggests that fractionation occurred at moderate pressure (probably $>15 \mathrm{~km}$ ) within the upper part of the lithospheric mantle. The presence of plagioclase phenocrysts and chemical evidence for plagioclase fractionation in the basalts with $\mathrm{MgO}<6$ is consistent with cooling and fractionation during storage at shallow depths.

Excellent agreement exists between different methods used to estimate a minimum pressure of melting for the Gran Canaria basalts. The inferred presence of residual garnet, phlogopite, and a carbonate phase argues for melting depths $\geqslant 3.0 \mathrm{GPa}$, consistent with the estimates from the $\mathrm{FeO}^{\prime}$ contents of the basalts $(>3.0 \mathrm{GPa})$ and from the basalt's CIPW normative compositions ( $\geqslant 3.5 \mathrm{GPa})$. As the mechanical boundary layer thickness beneath Gran Canaria is $\sim 100 \mathrm{~km}(\sim 3.0 \mathrm{GPa})$, all the Gran Canaria basalts probably originated by decompression melting of upwelling plume materal. The low melt production rate of the Canary Islands, about an order of magnitude lower than that for the Hawaiian Islands, can be explained by a thicker mechanical boundary layer (lithosphere) beneath the Canary 
Islands than beneath the Hawaiian Islands and does not necessarily require lower plume temperatures.

A number of models for the origin of the Canary Islands call upon a connection between volcanism and tectonic features on Africa or along fracture zones in the oceanic lithosphere [reviewed by Schmincke (1982)]. Although structural lineaments and other lines of weakness may play a role in channeling the magmas, depths of generation in excess of $100 \mathrm{~km}$ preclude these weaknesses from causing the volcanism unless the mechanical boundary layer beneath the Canary Islands is significantly thicker than $100 \mathrm{~km}$.

\section{ACKNOWLEDGEMENTS}

This study represents part of a Ph.D. dissertation [at University of California Santa Barbara (UCSB)] and postdoctoral study (at University of California Santa Cruz) by K.H. We are grateful to David Graham, George Tilton, Bill Wise, Brian Cousens, Frank Spera, Al Hofmann, and Jim Mattinson for comments on the manuscript and/or for stimulating discussions, and to $\mathrm{T}$. Wright and J. Ludden for official reviews of the manuscript. Special thanks go to George Tilton, without whose scientific and financial support this study would not have been possible. This research was in part supported by NSF grant EAR88-17802 to George Tilton and NSF grant EAR91-05113 to K.H., and by the Deutsche Forschungsgemeinschaft (Leibnitz grant) to H.-U.S. Henni Niephaus and Beate Schulz are thanked for their help with the XRF analyses, and Rodey Batiza, Pat Castillo, Jan Hertogen, and Bob Walker for providing us with INAA data. Funds for the INAA work at Oregon State University Radiation Center were provided by the US Department of Energy's Reactor Sharing Program, which we gratefully acknowledge. We thank Paul Robinson for allowing us to use his Miocene picrite and tholeiite samples. K.H. thanks the Deutscher Akademischer Austauschdienst (German Exchange Service) for a scholarship that funded his one and a half years in Bochum; they also helped fund his first field season on Gran Canaria. Grants from Mobil Oil, Sigma Xi, GSA, and the UCSB geology department were essential in helping fund two subsequent field seasons on Gran Canaria and two shorter visits to Bochum.

\section{REFERENCES}

Abdel-Monem, A., Watkins, N. D., \& Gast, P. W., 1971. Potassium-argon ages, volcanic stratigraphy, and geomagnetic polarity history of the Canary Islands: Lanzarote, Fuerteventura, Gran Canaria, and La Gomera. Am. J. ScI. 271, 490-521.

Albarede, F., \& Tamagnan, V., 1988. Modeling the recent geochemical evolution of the Piton de la Fournaise Volcano, Réunion Island, 1931-1986. J. Petrology 29, 997-1030.

Banda, E., Danobeitia, J. J., Surinach, E., \& Ansorge, J., 1981. Features of crustal structure under the Canary Islands. Earth Planet. Sci. Lett. 55, 11-24.

Brey, G., Brice, W. R., Ellis, D. J., Green, D. H., Harris, K. L., \& Ryabchikov, I. D., 1983. Pyroxene-carbonate reactions in the upper mantle. Earth Planet. Sci. Lett. 62, 63-74.

- Schmincke, H.-U., 1980. Origin and diagenesis of the Roque Nublo breccia, Gran Canaria (Canary Islands)petrology of Roque Nublo Volcanics II. Bull. Volcanol. 43, 15-33.

Clague, D. A., \& Dalrymple, G. B., 1988. Age and petrology of alkali postshield and rejuvenated-stage lava from Kauai, Hawaii. Contr. Miner. Petrol. 99, 202-18.

— Frey, F. A., 1982. Petrology and trace element geochemistry of the Honolulu Volcanics, Oahu: implications for the oceanic mantle below Hawaii. J. Petrology 23, 447-504. Weber, W. S., 1991. Picritic glasses from Hawaii. Nature 353, 553-6.

Cousens, B. L., 1990. Isotope geochemistry of lavas and pyroclastic flows of the Mogan and lower Fataga Formations, Gran Canaria, Canary Islands. Unpublished Ph.D. Thesis, University of California, Santa Barbara, 148 pp.

- Spera, F. J., \& Tilton, G. R., 1990. Isotopic patterns in silicic ignimbrites and lava flows of the Mogan and lower Fataga Formations, Gran Canaria, Canary Islands: temporal changes in mantle source composition. Earth Planet. Sci. Lett. 96, 319-35. 
Crisp, J. A., 1984. The Mogan and Fataga Formations of Gran Canaria (Canary Islands): geochemistry, petrology, and compositional zonation of the pyroclastic and lava flows; intensive thermodynamic variables within the magma chamber; and the depositional history of pyroclastic flow E/ET. Ph.D. Thesis, Princeton University, 304 pp.

_- Spera, F. J., 1987. Pyroclastic flows and lavas of the Mogan and Fataga formations, Tejeda Volcano, Gran Canaria, Canary Islands: mineral chemistry, intensive parameters, and magma chamber evolution. Contr. Miner. Petrol. 96, 503-18.

Falloon, T. J., \& Green, D. H., 1987. Anhydrous partial melting of MORB pyrolite and other peridotite compositions at $10 \mathrm{~kb}$ : implications for the origin of primitive MORB glasses. Miner. Petrol. 37, 181-219.

1988. Anhydrous partial melting of peridotite from 8 to $35 \mathrm{~kb}$ and the petrogenesis of MORB. J. Petrology, Special Lithosphere Issue, 279-414.

- Hatton, C. J., \& Harris, K. L., 1988. Anhydrous partial melting of a fertile and depleted peridotite from 2-30 kb and application to basalt petrogenesis. J. Petrology 29, 1257-82.

Feraud, G., Glannerini, G., Camprendon, R., \& Stillman, C. J., 1985. Geochronology of some Canarian dike swarms: contribution to the volcano-tectonic evolution of the archipelago. J. Volcanol. Geotherm. Res. 25, $29-52$.

—- Schmincke, H.-U., Lietz, J., Gastaud, J., Pritchard, G., \& Bleil, U., 1981. New K/Ar ages, chemical analyses and magnetic data of rocks from the islands of Santa Maria (Azores), Porto Santo and Madeira (Madeira Archipelago) and Gran Canaria (Canary Islands). Bull. Volcanol. 44, 359-75.

Fisk, M. R., Upton, B. J. G., Ford, C. E., \& White, W. M., 1988. Geochemical and experimental study of the genesis of magmas of Réunion Island, Indian Ocean. J. Geophys. Res. 93, 4933-50.

Flower, M. F. J., Pritchard, R. G., Schmincke, H.-U., \& Robinson, P. T., 1983. Geochemistry of basalts: Deep Sea Drilling Project sites 482, 483, and 485 near the Tamayo fracture zone, Gulf of California. In: Initial Reports of the Deep Sea Drilling Project. Washington, DC: US Government Printing Office, 559-78.

Frey, F. A., Green, D. H., \& Roy, S. D., 1978. Integrated models of basalt petrogenesis: a study of quartz tholeiites to olivine melilitites from South Eastern Australia. J. Petrology 19, 463-513.

Green, D. H., Falloon, T. J., \& Taylor, W. R., 1987. Mantle derived magmas-role of variable source peridotite and variable C-H-O fluid compositıons. In: Mysen, B. O. (ed.) Magmatic Processes: Physiochemical Principles. Geochem. Soc. Spec. Publ. 1, 139-54.

Hanson, G. N., 1980. Rare earth elements in petrogenetic studies of igneous systems. Ann. Rev. Earth Planet. Sci.8, $371-406$

Hawkesworth, C. J., Kempton, P. D., Rogers, N. W., Ellam, R. M., \& van Calsteren, P. W., 1990. Continental mantle lithosphere, and shallow level enrichment processes in the Earth's mantle. Earth Planet. Sci. Lett. 96, $256-68$.

Hertogen, J., \& Gijbels, R., 1971. Instrumental neutron activation analysis of silicate rocks with a low-energy photon detector. Analyt. Chim. Acta 56, 61-82.

Hoernle, K. A., 1987. General geology and petrology of the Roque Nublo Volcanics on Gran Canaria, Canary Islands, Spain. Unpublished M.A. Thesis, Unuversity of California, Santa Barbara, 191 pp.

— Tilton, G., \& Schmincke, H.-U., 1991. Sr-Nd-Pb isotopic evolution of Gran Canaria: evidence for shallow enriched mantle beneath the Canary Islands. Earth Planet. Sci. Lett. 106, 44-63.

Irving, A. J., \& Frey, F. A., 1984. Trace element abundances in megacrysts and their host basalts: constraints on partition coefficients and megacryst genesis. Geochim. Cosmochim. Acta 48, 1201-21.

Jacobs, J. W., Korotev, R. L., Blanchard, D. P., \& Haskin, L. A., 1977. A well-tested procedure for instrumental neutron activation analysis of silicate rocks and minerals. J. Radioanal. Chem. 40(1-2), 93-114.

Jacques, A. L., \& Green, D. H., 1980. Anhydrous melting of peridotite at $0-15 \mathrm{kbar}$ pressure and the genesis of tholeiitic basalts. Contr. Miner. Petrol 73, 287-310.

Klein, E. M., \& Langmuir, C. H., 1987. Global correlations of ocean ridge basalt chemistry with axial depth and crustal thickness. J. Geophys. Res. 92, 8089-115.

Langmuir, C. H., \& Hanson, G. N., 1980. An evaluation of major element heterogeneity in the mantle sources of basalts. Phsl. Trans. R. Soc. Lond. A297, 383-407.

Laul, J. C., \& Schmitt, R. A., 1973. Chemical composition of Luna 20 rocks and soll and Apollo 16 soils. Geochim. Cosmochim. Acta 37, 927-42.

Le Bas, M. J., Le Maitre, R. W., Streckheisen, A., \& Zanettin, B., 1986a. A chemical classification of volcanic rocks based on the total alkali-silica diagram. J. Petrology 27, 745-50.

— Rex, D. C., \& Stillman, C. J., 1986b. The early magmatic chronology of Fuerteventura, Canary Islands. Geol. Mag. 123(3), 287-98.

Lietz, J., \& Schmincke, H.-U., 1975. Miocene-Pliocene sea level changes and volcanic episodes on Gran Canaria (Canary Islands) in the light of new K/Ar ages. Palaeogeogr. Palaeoclimatol. Palaeoecol. 18, 213-39.

Lindstrom, D. J., \& Korotev, R. L., 1983. TEABAGS: computer programs for instrumental neutron activation analysis. J. Radioanal. Chem. 70(1-2), 439-58.

Macdonald, G. A., \& Katsura, T., 1964. Chemical composition of Hawaiian lavas. J. Petrology 5, 82-133.

Mahood, G. C., \& Baker, D. R., 1986. Experimental constraints on depths of fractionation of mildly alkalic basalts and associated felsic rocks: Pantelleria, Stratt of Sicily. Contr. Miner. Petrol. 93, 251-64.

Mankinen, E. A., \& Dalrymple, G. B., 1979. Revised geomagnetic polarity time scale for the interval 0-5 m.y. B.P.J. Geophys. Res. 84, 615-26. 
McDougall, I., \& Schmincke, H.-U., 1976. Geochronology of Gran Canaria, Canary Islands: age of shield building volcanism and other magmatic phases. Bull. Volcanol. 40,1-21.

McKenzie, D., \& Bickle, M. J., 1988. The volume and composition of melt generated by extension of the lithosphere. J. Petrology 29, 625-79.

O'Nions, R. K., 1991. Partial melt distributions from inversion of rare earth element concentrations. Ibid. 32, 1021-91.

Peterson, T. D., 1989. Peralkaline nephelintes. 1. Comparative petrology of Shombole and Oldoinyo L'enga1, East Africa. Contr. Miner. Petrol. 101, 458-78.

Schmincke, H.-U., 1973. Magmatic evolution and tectonic regime in the Canary, Madeira and Azores Island Groups. Geol. Soc. Am. Bull. 84, 633-48.

1976. The geology of the Canary Islands. In: Kunkel, G. (ed.) Biogeography and Ecology of the Canary Islands. The Hague: Junk, 67-184.

- 1982. Volcanic and chemical evolution of the Canary Islands. In: von Rad, U., Hinz, K., Sarnthein, M., \& Seibold, E. (eds.) Geology of the Northwest African Margin. New York: Springer-Verlag, 273-306. 1987-1990. Geological Field Guide of Gran Canaria. Witten, Germany: Pluto Press, 4 editions.

_ Duncan, R., Balcells, R., Barrera, J. L., \& Gomes, J. A. (in prep.). Temporal and chemical evolution of Gran Canaria.

- Flower, M. F. J., 1974. Magmenevolution auf Atlantischen Vulkaninseln. Naturwissenschaften 61, $288-92$.

Sleep, N. H., 1990. Hotspots and mantle plumes: some phenomenology. J. Geophys. Res. 95, 6715-36.

Sun, S.-S., \& McDonough, W. F., 1989. Chemical and isotopic systematics of oceanic basalts: implications for mantle composition and processes. In Saunders, A. D., \& Norry, M. J. (eds.) Magmatism in the Ocean Basins. Geol. Soc. Spec. Publ. 42, 313-45.

Takahashi, E., 1986. Melting of a dry peridotite KLB-1 up to $14 \mathrm{GPa}$ implications on the origin of periodotitic upper mantle. J. Geophys. Res. 91, 9367-82.

Kushiro, I., 1983. Melting of a dry peridotite at high pressures and basalt magma genesis. Am. Miner. 68, 859-79.

van den Bogaard, P., Schmincke, H.-U., Freundt, A., Hall, C. M., \& York, D., 1988. Eruption ages and magma supply rates during the Miocene evolution of Gran Canaria. Naturwissenschaften 25, 616-17.

Watson, E. B., 1979. Zırcon saturation in felsic liquids: experimental results and applications to trace element geochemistry. Contr. Miner. Petrol. 70, 407-19.

- Harrison, T. M., 1983. Zircon saturation revisited: temperature and composition effects in a variety of crustal magma types. Earth Planet. Sci. Lett. 64, 295-304.

Watson, S., \& McKenzie, D., 1991. Melt generation by plumes: a study of Hawaiian volcanism. J. Petrology 32, $501-37$.

Wright, T. L., 1984. Origin of Hawaiian tholeiite: a metasomatic model. J. Geophys. Res. 89, 3233-52.

Zhang, Y.-S., \& Tanimoto, T., 1991. Global Love wave phase velocity variation and its significance to plate tectonics. Phys. Earth Planet. Inter. 66, 160-202. 